United States Department of Commerce Technology Administration

National Institute of Standards and Technology

NISTIR 3981

\title{
SIMULATION OF THE MERGED SPECTRUM TECHNIQUE FOR ALIGNING PLANAR PHASED-ARRAY ANTENNAS, PART I
}

Ronald C. Wittmann

Allen C. Newell

Carl F. Stubenrauch

Katherine MacReynolds

Michael H. Francis 



\section{SIMULATION OF THE MERGED SPECTRUM TECHNIQUE FOR ALIGNING PLANAR PHASED-ARRAY ANTENNAS, PART I}

Ronald C. Wittmann

Allen C. Newell

Carl F. Stubenrauch

Katherine MacReynolds

Michael H. Francis

Electromagnetic Fields Division

Electronics and Electrical Engineering Laboratory

National Institute of Standards and Technology

Boulder, Colorado 80303-3328

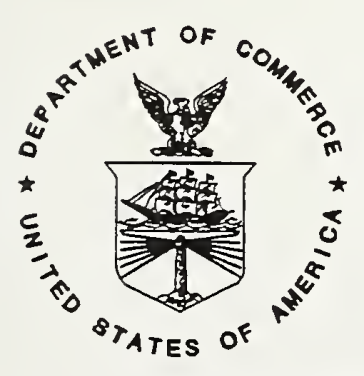

U.S. DEPARTMENT OF COMMERCE, Barbara Hackman Franklin, Secretary TECHNOLOGY ADMINISTRATION, Robert M. White, Under Secretary for Technology NATIONAL INSTITUTE OF STANDARDS AND TECHNOLOGY, John W. Lyons, Director 


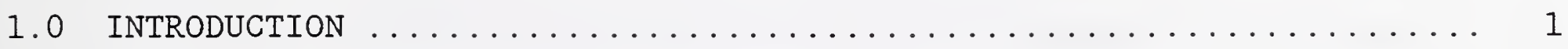

2.0 BASIC NEAR-FIELD MEASUREMENT THEORY $\ldots \ldots \ldots \ldots \ldots \ldots \ldots \ldots \ldots \ldots \ldots \ldots$

2.1 The Spectral Representation of Radiated Fields ............. 2

2.2 Kerns's Transmission Equation ..................... 3

2.3 Inverting the Transmission Equation ................. 4

2.4 Sampling the Near Field ....................... 4

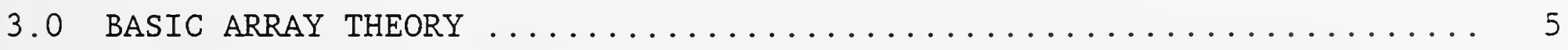

3.1 The Fields of a Planar Antenna Array ................. 5

3.2 The Array Factor for a Broadside Beam .................. 7

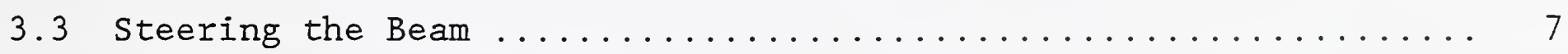

3.4 The Array Factor for a Regular Lattice ................ 8

3.5 Transforming Between Array Factor and Excitation Matrix ....... 10

4.0 THE MERGED-SPECTRUM TECHNIQUE ...................... 12

4.1 Choosing Parameters .......................... 12

4.2 Determining the Form Factor from Near-Field Data ........... 13

4.3 Combining Beams ............................. 14

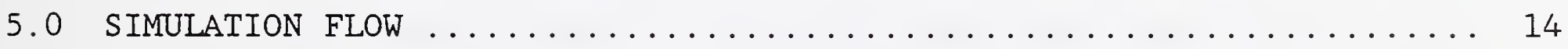

5.1 Array Mode1 ............................. 14

5.2 Simulation Examples ........................ 15

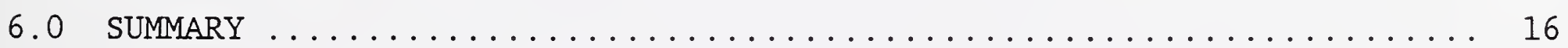

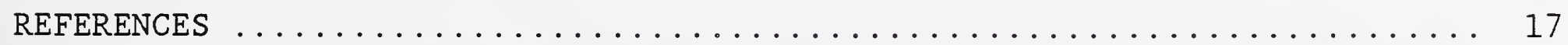


1. A centered rectangular lattice showing a set of fundamental (and primitive) lattice vectors. $a=\lambda(1 / 2 \mathrm{x}-3 / 10 \mathrm{y}), \mathrm{b}=\lambda 3 / 5 \mathrm{y}$.

2. The reciprocal lattice for the lattice of figure $1 . A=2 k \hat{x}$, $B=k(x+5 / 3 y)$. If the array factor is known on the fundamental period (shaded), then it may be determined everywhere by periodic repetition. For example, in the open rectangle $\mathrm{A}(\mathbf{K})=\mathrm{A}(\mathbf{K}-\mathbf{B}) \ldots \ldots 18$

3. A rectangular lattice obtained from the centered rectangular lattice of figure 1 by adding interstitial points. $a^{\prime}=\lambda 1 / 2 \mathrm{x}$, $\mathrm{b}^{\prime}=\lambda 3 / 10 \mathrm{y} . \quad$ Element positions are marked with $\mathrm{x}^{\prime} \mathrm{s} . \quad\left(\alpha_{\mathrm{nm}}^{\prime}=0, \mathrm{n}+\mathrm{m}\right.$

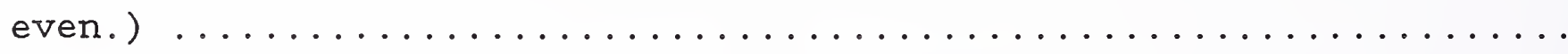

4. A fundamental period in the reciprocal lattice for the doubled lattice of figure 3. A(K) may be determined everywhere from its values on the fundamental period of the centered rectangular sublattice (shaded). The mapping is indicated by quadrant. The minus signs occur because the centered rectangular sublattice is offset from the origin.

5. The visible region for a broadside beam is the shaded disk. Extrapolation would be necessary to determine the form factor from this information. In the merged-spectrum technique, direct measurement of the fundamental period (rectangle) is accomplished via beam steering.

6. When the beam is steered to the four locations $k_{0}=k( \pm 1 / 2 \hat{\mathbf{x}} \pm 5 / 12 \hat{y})$ marked with ''s, the entire fundamental period can be measured directly. The visible region corresponding to each beam direction is indicated by a circle. The shaded rectangle $\left(\theta \leq 40.6^{\circ}\right)$ is the smallest region in which coupling products must be determined.

7. Block diagram showing the phased-array simulation and the near-field measurement simulation. 


\section{LIST OF FIGURES (continued)}

Figure

8. Block diagram showing the merged-spectrum technique for determining

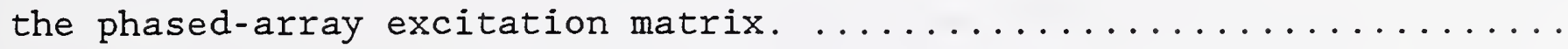

9. Schematic of the phased array showing location of active elements...

10. Schematic of the central part of the phased array showing location of active elements and virtual elements.

11. Relative amplitude excitations (dots) of elements along the center row, $\mathrm{y}=0$, and field amplitude (solid line), normalized to $0 \mathrm{~dB}$ peak, at $z=20 \mathrm{~cm}$. Frequency is $3 \mathrm{GHz}$.

12. Relative amplitude excitations (dots) of elements along the center column, $\mathrm{x}=0$, and field amplitude (solid line), normalized to $0 \mathrm{~dB}$ peak, at $z=20 \mathrm{~cm}$. Frequency is $3 \mathrm{GHz}$.

13. Relative amplitude excitations (dots) of elements along the center row, $\mathrm{y}=0$, and field amplitude (solid line), normalized to $0 \mathrm{~dB}$ peak, at $z=50 \mathrm{~cm}$. Frequency is $3 \mathrm{GHz}$.

14. Relative amplitude excitations (dots) of elements along the center column, $\mathrm{x}=0$, and field amplitude (solid line), normalized to $0 \mathrm{~dB}$ peak, at $z=50 \mathrm{~cm}$. Frequency is $3 \mathrm{GHz}$

15. Contour plot of the array factor for the doubled array composed of

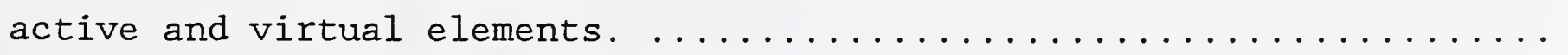

16. Perspective plot of the array factor for the doubled array composed

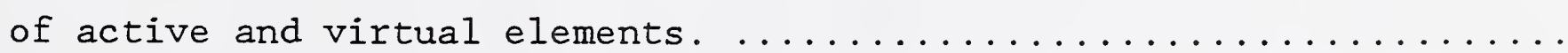

17. Principal plane $\left(k_{y}=0\right)$ cut of the array factor for the doubled array composed of active and virtual elements. 
18. Principal plane $\left(k_{x}=0\right)$ cut of the array factor for the doubled

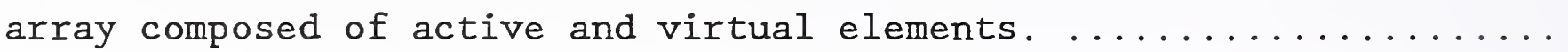

19. Contour plot of the probe's receiving pattern, amplitude for openended waveguide.

20. Contour plot of the probe's receiving pattern, phase for open-ended waveguide.

21. Amplitude of the coupling product $D^{e}(\hat{\mathbf{k}})$ assuming probe and average element are open-ended waveguides.

22. Phase of the factor $\exp (i \gamma z)$. Frequency is $3 \mathrm{GHz}$ and $z=20 \mathrm{~cm} . \ldots$

23. Amplitude of the factor $\exp (i \gamma z)$. Frequency is $3 \mathrm{GHz}$ and $z=20 \mathrm{~cm}$.

24. Simulated near-field amplitude. Frequency is $3 \mathrm{GHz}$ and $z=20 \mathrm{~cm}$. .

25. Perspective plot of the near-field amplitude. Frequency is $3 \mathrm{GHz}$ and $z=20 \mathrm{~cm}$.

26. High resolution contour plot of the near-field amplitude near the center of the aperture. Frequency is $3 \mathrm{GHz}$ and $z=50 \mathrm{~cm}$.......

27. High resolution contour plot of the near-field phase near the center of the aperture. Frequency is $3 \mathrm{GHz}$ and $z=50 \mathrm{~cm}$.

28. Contour plot of the array factor for an excitation matrix with sinusoidal errors in both $\mathrm{x}$ and $\mathrm{y}$ directions $\left(3^{\circ}\right.$ amplitude, $15 \mathrm{~cm}$ period). 
29. Perspective plot of the array factor for an excitation matrix with sinusoidal errors in both $\mathrm{x}$ and $\mathrm{y}$ directions $\left(3^{\circ}\right.$ amplitude, $15 \mathrm{~cm}$ period).

30. Principal plane $\left(k_{y}=0\right)$ cut of the array factor for an excitation matrix with a sinusoidal phase error in the $\mathrm{x}$ direction ( $3^{\circ}$ amplitude, $15 \mathrm{~cm}$ period).

31. Principal plane $\left(k_{x}=0\right)$ cut of the array factor for an excitation matrix with a sinusoidal phase error in the y direction ( $3^{\circ}$ amplitude, $15 \mathrm{~cm}$ period).

32. Contour plot of array factor for a steered beam. .............. 42 



\title{
SIMULATION OF THE MERGED-SPECTRUM TECHNIQUE \\ FOR ALIGNING PLANAR PHASED-ARRAY ANTENNAS, \\ PART I
}

\author{
Ronald C. Wittmann \\ Allen C. Newell \\ Carl F. Stubenrauch \\ Katherine MacReynolds \\ Michael H. Francis
}

\section{Electromagnetic Fields Division}

National Institute of Standards and Technology

Boulder, CO 80303-3328

\begin{abstract}
This report describes the initial phase of a NIST study of the merged-spectrum technique for determining the element excitations from planar scanning near-field measurements of phased-array antennas. Excitation data are used in adjusting phase shifters to meet design specifications. Measurement uncertainties, steering errors, and various analytic approximations will all introduce errors into the alignment. The study is ultimately directed to quantify the effect of these errors, to more fully understand the merged-spectrum technique, and to recommend possible improvements. The present report covers theory developed to support evaluation of the merged-spectrum technique and gives simulation examples illustrating calculation of near fields from array factor and element patterns.
\end{abstract}

Key words: antenna measurements; antenna array alignment; merged spectrum; near-field measurements; phased-array antennas; planar scanning.

\subsection{INTRODUCTION}

This report describes the initial phase of a NIST study of a method for determining the element excitations (amplitude and phase) from planar nearfield measurements of phased-array antennas. The method is called the mergedspectrum technique because the (broadside-beam) array factor is obtained from several spectra, each measured with the array electrically steered to a different direction [1]. (There may not be enough information in any one spectrum to determine the array factor directly.) 
Excitation data are used in adjusting phase shifters, which initially may be set randomly, to meet design specifications. Measurement uncertainties, steering errors, and various analytic approximations will all introduce errors into the alignment. The study is ultimately directed to quantify the effect of these errors, to more fully understand the merged-spectrum technique, and to recommend possible improvements. The present report covers computer code and theory developed to support evaluation of the merged-spectrum technique.

A first step, contained in sections 2 through 4, is a careful description of measurement and theory needed for the merged-spectrum technique. Section 2 is a brief summary of the principles, relations, and notation of the planar near-field scanning measurement technique. In section 3 phased-array antennas are introduced and the concepts of element excitations, average element pattern, and array factor are discussed. The relations between the lattice of array elements and the corresponding reciprocal lattice in $\mathrm{k}$ space are developed since these are critical concepts in the merged-spectrum technique. Section 4 sketches the merged-spectrum theory. In section 5 we give some simulation results, illustrating calculation of array factors for steered and unsteered beams, as well as simulated near-field measurements for typical element and probe patterns. Additionally, examples of the effect of certain measurement errors are shown.

\subsection{BASIC NEAR-FIELD MEASUREMIENT THEORY}

The theory of planar near-field scanning is briefly summarized in this section. A thorough account may be found in Kerns [2].

\subsection{The Spectral Representation of Radiated Fields}

The theory of planar near-field scanning is based on the fact that radiated fields can be written as a superposition of plane waves

$$
\mathbf{E}(\mathbf{r})=\frac{1}{2 \pi} \int t(\hat{\mathbf{k}}) \exp (\mathbf{i k} \cdot \mathbf{r}) \frac{\mathrm{dK}}{\gamma \mathrm{k}}, \quad z>0
$$

where an $\exp (-i \omega t)$ time dependence is suppressed. Here

$$
\mathbf{k}=\mathbf{k}+\hat{\gamma \mathbf{z}}, \quad \mathbf{k}=\mathrm{k}_{\mathrm{x}} \hat{\mathbf{x}}+\mathrm{k}_{\mathrm{y}} \hat{\mathbf{y}}, \quad \mathrm{K}=|\mathbf{K}|
$$




$$
\begin{aligned}
& \gamma=k_{z}= \begin{cases}\sqrt{k^{2}-k^{2}}, & k \geq k \\
i \sqrt{k^{2}-k^{2}}, & k<k\end{cases} \\
& \int d K \equiv \int_{-\infty}^{\infty} d k_{x} \int_{-\infty}^{\infty} d k y .
\end{aligned}
$$

The far-field pattern $t(\hat{r})$ is defined so that

$$
E(r) \underset{r \longrightarrow \infty}{\sim} t(\hat{r}) \frac{\exp (i k r)}{i k r}
$$

The quantity $\mathbf{t}(\hat{\mathbf{k}}) / \gamma \mathrm{k}$ is called the spectrum of $\mathrm{E}(\mathbf{r})$.

\subsection{Kerns's Transmission Equation}

The output of a probe can be related to the incident field through the transmission equation

$$
\begin{aligned}
W(\mathbf{r}) & =\int \mathbf{r}(\hat{\mathbf{k}}) \cdot \mathbf{t}(\hat{\mathbf{k}}) \exp (\mathbf{i} \mathbf{k} \cdot \mathbf{r}) \frac{\mathrm{dK}}{\gamma \mathbf{k}} \\
& =\int \mathrm{D}(\hat{\mathbf{k}}) \exp (\mathbf{i} \gamma z) \exp (\mathbf{i} \mathbf{K}) \mathrm{r} \mathbf{K}, \quad z>0 \\
& D(\hat{\mathbf{k}}) \equiv \mathbf{r}(\hat{\mathbf{k}}) \cdot \boldsymbol{t}(\hat{\mathbf{k}}) / \gamma \mathbf{k} .
\end{aligned}
$$

$D(\hat{k})$ is the coupling product. The receiving pattern $\mathbf{r}(\mathbf{k})$ is defined so that the response of the probe to the incident plane wave

$$
\frac{I}{2 \pi} \mathbf{E}_{0} \exp (\mathbf{i k} \cdot \mathbf{r})
$$

is

$$
\mathbf{r}(\hat{\mathbf{k}}) \cdot \mathbf{E}_{0} \exp \left(\mathbf{i k} \cdot \mathbf{r}_{\mathrm{p}}\right)
$$

where $r_{p}$ is the position of the probe. The definition of the receiving pattern depends on the choice of probe calibration; however, the details of this normalization are unimportant for the present application. 
Basically, the transmission equation states that the probe output is the sum of the probe responses to the plane-wave components of the incident field eq (1).

\subsection{Inverting the Transmission Equation}

Fourier transformation of eq (3) gives

$$
D(\hat{\mathbf{k}})=\frac{1}{4 \pi^{2}} \int \mathrm{W}(\mathbf{r}) \exp (-\mathbf{i k} \cdot \mathbf{r}) \mathrm{d} \mathbf{R}, \quad z>0
$$

or

$$
\begin{aligned}
& D(\hat{\mathbf{k}}) \exp \left(\hat{i} \gamma z_{0}\right)=\frac{1}{4 \pi^{2}} \int W(\mathbf{r}) \exp (-\mathbf{i} \mathbf{R} \cdot \mathbf{R}) \mathrm{dR}, \quad z>0 \\
& \mathbf{r}=\mathbf{R}+z_{0} \hat{z}, \quad \mathbf{R}=\hat{x} \mathbf{x}+\hat{y} \mathbf{Y} \\
& \int \mathrm{d} \mathbf{R} \equiv \int_{-\infty}^{\infty} \mathrm{dx} \int_{-\infty}^{\infty} \mathrm{dy} .
\end{aligned}
$$

Thus, in principle, the coupling product may be determined if the probe response is known on a plane $z=z_{0}>0$.

\subsection{Sampling the Near Field}

The application of eq (4) is greatly simplified by the fact that $D(\hat{\mathbf{k}})$ is strongly spatially bandlimited because the plane-wave components become evanescent when $\mathrm{K}>\mathrm{k}$. Thus, according to the "sampling theorem," the coupling product may be reconstructed "exactly" from discrete probe measurements as long as the sample interval is less than half a wavelength:

$$
\Delta \mathrm{x}, \Delta \mathrm{y} \leq \lambda / 2
$$

In an actual measurement, of course, the scan area must be finite. A rule of thumb is that every line drawn from the aperture in a direction in which the far field is to be determined must pass through the measurement region. (Like all rules of thumb, this is subject to experimental verification.) 
With the assumption of discrete sampling and truncation of the integration range, eq (4) may be reduced to a DFT (Discrete Fourier Transform). By the nature of the DFT (discussed more fully in section 3.5), measurements made at the points

$$
r_{n m}=n \Delta x \hat{x}+m \Delta y \hat{y}+z_{0} \hat{z}
$$

result in the determination of $D(\hat{\mathbf{k}})$ for

$$
\mathrm{K}_{\mathrm{nm}}=\frac{\mathrm{n}}{\overline{\mathrm{N}}_{\mathrm{x}}} \frac{2 \pi}{\Delta \mathrm{x}} \hat{\mathrm{x}}+\frac{\mathrm{m}}{\overline{\mathrm{N}}_{\mathrm{y}}} \frac{2 \pi}{\Delta \mathrm{y}} \hat{\mathrm{y}},
$$

where $\mathrm{N}_{\mathrm{x}}$ and $\mathrm{N}_{\mathrm{y}}$ are the numbers of data points in the $\mathrm{x}$ and $\mathrm{y}$ directions. The $\mathrm{K}$ space increments $\Delta \mathrm{k}_{\mathrm{x}}$ and $\Delta \mathrm{k}_{\mathrm{y}}$ are inversely proportional to the $\mathrm{x}$ and $\mathrm{y}$ dimensions of the measurement plane.

It is often useful to zero-fill the data array by explicitly (rather than implicitly) setting the probe response to 0 outside the actual measurement region. This does not add any information, but it does result in evaluation of the coupling product on a finer grid [ since $\mathrm{N}_{\mathrm{x}}$ and/or $\mathrm{N}_{\mathrm{y}}$ have been increased in eq (6)]. Also, the FFT (Fast Fourier Transform) algorithm used to perform the DFT is most efficient if $\mathrm{N}_{\mathrm{x}}$ and $\mathrm{N}_{\mathrm{y}}$ can be factored into small primes. For example, use $\mathrm{N}_{\mathrm{x}}=256$, but not $\mathrm{N}_{\mathrm{x}}=251$.

\subsection{BASIC ARRAY THEORY}

This section describes the basic elements of antenna array theory needed for an understanding of the merged-spectrum technique. A good general reference is $\mathrm{Ma}$ [3].

\subsection{The Fields of a Planar Antenna Array}

Consider a planar array made up of identical elements. The electric field of the array is a superposition of the element fields:

$$
\mathbf{E}(\mathbf{r})=\sum_{i} a\left(\mathbf{R}_{\mathbf{i}}\right) \mathbf{e}\left(\mathbf{r}-\mathbf{R}_{\mathbf{i}}\right)
$$

The sum runs over the positions of the array elements $\mathbf{R}_{\mathbf{i}}$. We take 


$$
\mathbf{R}_{i}=x_{i} \hat{x}+y_{i} \hat{y}
$$

so that the array lies in the $z=0$ plane. The excitation matrix $a\left(\mathbf{R}_{i}\right)$ determines the amplitude and phase of the element excitations. The beam is shaped and steered by controlling $a\left(\mathbf{R}_{i}\right)$. The average element field $\mathbf{e}(\mathbf{r})$ is not the free-space field of an element, but includes effects of mutual interactions among elements and supports, etc. (In a finite array it is an approximation to assume that all elements produce the same effective field since the environment varies from location to location.)

An array element is characterized by a far-field pattern $t^{e}(\hat{\mathbf{k}})$, which we define so that

$$
e(r) \underset{r \longrightarrow \infty}{\sim} t^{e} \hat{(r)} \frac{\exp (i k r)}{i k r}
$$

When an element is positioned in the array, its far-field pattern is multiplied by a phase factor depending on the displacement from the coordinate origin (which is the mathematical "location" of the array):

$$
\mathbf{e}\left(\mathbf{r}-\mathbf{R}_{\mathbf{i}}\right) \underset{r \longrightarrow \infty}{\sim} t^{e} \hat{(\mathbf{r})} \exp \left(-\mathbf{i k \hat { r }} \cdot \mathbf{R}_{i}\right) \frac{\exp (i k r)}{i k r}
$$

From eqs (7) and (10)

$$
\mathbf{E}(\mathbf{r}) \underset{r \longrightarrow \infty}{\sim} t^{a} \hat{(\mathbf{r})} \frac{\exp (i k r)}{i k r}
$$

where $t^{a}(\hat{r})$ is given by

$$
\begin{aligned}
\left.t^{a} \hat{(\mathbf{r}}\right) & =t^{e} \hat{(\mathbf{r})} \sum_{i} a\left(\mathbf{R}_{i}\right) \exp \left(-i \hat{i k r} \cdot \mathbf{R}_{i}\right) \\
& \equiv t^{e} \hat{(\mathbf{r})} A(k \mathbf{R} / r) .
\end{aligned}
$$

The far-field pattern of the array is the product of the average element farfield pattern and the array factor $A(\mathbf{r})$.

According to eq (1), the electric field of the array may be written in terms of its far-field pattern: 


$$
\begin{aligned}
& \mathbf{E}(\mathbf{r})=\frac{1}{2 \pi} \int \mathbf{t}^{\mathrm{a}} \hat{\hat{\mathbf{k}})} \exp (\mathbf{i k} \cdot \mathbf{r}) \frac{\mathrm{d} \mathbf{K}}{\gamma \mathbf{k}} \\
& =\frac{1}{2 \pi} \int t^{e}(\hat{\mathbf{k}}) \mathrm{A}(\mathbf{K}) \exp (\mathbf{i k} \cdot \mathbf{r}) \frac{\mathrm{d} \mathbf{K}}{\gamma \mathbf{k}}, \quad z>0 \\
& A(\mathbf{K})=\sum_{i} a\left(\mathbf{R}_{\mathbf{i}}\right) \exp \left(-\mathbf{i} \mathbf{K} \cdot \mathbf{R}_{\mathbf{i}}\right)
\end{aligned}
$$

\subsection{The Array Factor for a Broadside Beam}

When the beam is broadside, the $a\left(\mathbf{R}_{i}\right)$ must have constant phase since this will maximize the far-field pattern in the $z$ direction: If the broadside-beam excitation matrix and the corresponding array factor are denoted by $\alpha\left(\mathbf{R}_{i}\right)$ and $A_{0}(K)$, respectively, then from eq (14)

$$
\left|A_{0}(0)\right|=\sum_{i}\left|\alpha\left(R_{i}\right)\right| \geq\left|A_{0}(K)\right|
$$

The purpose of the merged-spectrum alignment is to determine the actual values of the $\alpha\left(\mathbf{R}_{i}\right)$ for an unaligned array so they may be adjusted to the design values. We will call $A_{0}(\mathbf{K})$ the form factor of the array. As demonstrated in section 3.3, the array factor of a steered beam is simply related to the form factor.

\subsection{Steering the Beam}

Steering is accomplished by adjusting the phase of the excitation matrix. When the excitation matrix is

$$
a\left(\mathbf{R}_{i}\right)=\alpha\left(\mathbf{R}_{i}\right) \exp \left(i \mathbf{R}_{0} \cdot \mathbf{R}_{i}\right)
$$

then the array factor

$$
A(\mathbf{K})=A_{0}\left(\mathbf{K}-\mathbf{K}_{0}\right)=\sum_{i} \alpha\left(\mathbf{R}_{i}\right) \exp \left[-i\left(\mathbf{K}-\mathbf{K}_{0}\right) \cdot \mathbf{R}_{i}\right]
$$

will produce a beam in the $\hat{\mathbf{k}}_{0}$ direction. With the convention eq (16), the phase of a reference element at the origin will be independent of $\mathbf{k}_{0}$. 
The electric field of the steered array is

$$
\mathbf{E}(\mathbf{r})=\frac{1}{2 \pi} \int t^{e}(\hat{\mathbf{k}}) \mathrm{A}_{0}\left(\mathbf{K}-\mathbf{K}_{0}\right) \exp (\mathbf{i k} \cdot \mathbf{r}) \frac{\mathrm{d} \mathbf{K}}{\gamma \mathbf{k}}, \quad z>0
$$

Since the plane-wave components of eq (18) become evanescent when $\mathrm{K}>\mathrm{k}, \mathrm{A}_{0}$ (L) plays an active role in determining the far field of the array only when $\mathbf{L}$ lies within a circle of radius $k=\omega / c$ centered on $\mathbf{L}=-\mathbf{K}_{0}$. Thus, the visible portion of the form factor depends on the steering angle.

The steering vector $\mathbf{k}_{0}$ can be related to the usual polar angles $\theta$ and $\phi$ :

$$
\begin{array}{ll}
\mathrm{K}_{0 \mathrm{x}}=\mathrm{k} \sin \theta \cos \phi, & \mathrm{K}_{0 \mathrm{y}}=\mathrm{k} \sin \theta \sin \phi \\
\sin \theta=\mathrm{K}_{0} / \mathrm{k}, & \tan \phi=\mathrm{K}_{0 \mathrm{y}} / \mathrm{K}_{0 \mathrm{x}} .
\end{array}
$$

\subsection{The Array Factor for a Regular Lattice}

Every point in a planar lattice can be represented in the form

$$
\mathbf{R}_{\mathrm{nm}}=\mathrm{na}+\mathrm{mb}
$$

$(\mathbf{a} \times \mathbf{b} \neq 0)$. The fundamental lattice vectors $\mathbf{a}$ and $\mathbf{b}$ are said to be primitive if $\mathbf{R}_{\mathrm{nm}}$ is a lattice point for all integers $\mathrm{n}$ and $\mathrm{m}$.

In this report we will assume that the array elements lie on a centered rectangular lattice, illustrated in figure 1 , where the fundamental lattice vectors are given by

$$
\mathbf{a}=\lambda\left(\frac{1}{2} \hat{\mathbf{x}}-\frac{3}{10} \hat{\mathbf{y}}\right), \quad \mathbf{b}=\frac{3}{5} \lambda \hat{\mathbf{y}}
$$

$\mathbf{a}$ and $\mathbf{b}$ are clearly primitive. [The choice of $\mathbf{a}$ and $\mathbf{b}$ is not unique; for example, we could use $\left.\mathbf{a}=\lambda\left(\frac{1}{2} \hat{\mathbf{x}}+\frac{3}{10} \hat{\mathbf{y}}\right).\right]$

The array factor eq (14) may now be written

$$
\begin{aligned}
& A(\mathbf{R})=\sum_{\nu \mu} a_{\nu \mu} \exp \left(-i \mathbf{R} \cdot \mathbf{R}_{\nu \mu}\right) \\
& a_{\mathrm{nm}} \equiv a\left(\mathbf{R}_{\mathrm{nm}}\right) .
\end{aligned}
$$

It is easy to show from eq (21) that the array factor is doubly periodic 


$$
A(K \pm A)=A(K), \quad A(K \pm B)=A(K)
$$

where

$$
\mathbf{A} \equiv 2 \pi \frac{\mathbf{b} \times \hat{\mathbf{z}}}{|\mathbf{a} \times \mathbf{b}|}=2 \mathrm{k} \hat{\mathbf{x}}, \quad \mathbf{B} \equiv 2 \pi \frac{\hat{\mathbf{z}} \times \mathbf{a}}{|\mathbf{a} \times \mathbf{b}|}=\mathrm{k}\left(\hat{\mathbf{x}}+\frac{5}{3} \hat{\mathbf{y}}\right)
$$

[For a rectangular lattice with $\mathbf{a}=\hat{a} \mathbf{x}, \mathbf{b}=\hat{b y}$, we have $\mathbf{A}=2 \pi / \mathbf{a} \hat{\mathbf{x}}$, $\mathbf{B}=2 \pi / \mathrm{b} \mathbf{y}$. In general, $|\mathbf{A}|$ is proportional to $1 / \mathrm{a}$ (independent of $\mathrm{b}$ ) and $|\mathbf{B}|$ is proportional to $1 / b$ (independent of $a$ ).]

$\mathbf{A}$ and $\mathbf{B}$ are fundamental lattice vectors of the reciprocal lattice shown in figure 2. The form factor $A_{0}(K)$ assumes its maximum amplitude at each lattice point $\left(\mathbf{K}_{\mathrm{nm}}=\mathrm{n} \mathbf{A}+\mathbf{m B}\right)$. Consequently, if more than one reciprocal lattice point is visible, then, in addition to the main beam, there may be other beams (grating lobes) in the far-field pattern. Figure 2 also shows a fundamental period (shaded rectangle) for the array factor. If the array factor is known on a fundamental period then it may be determined everywhere by periodic repetition using eq (22). (While the area is fixed, a fundamental period may assume a number of shapes. A rectangle is convenient for our purposes.)

The preceding discussion assumes that $\mathbf{R}=0$ is a lattice point (that is, there is an element at the origin). If the array is offset so that

$$
\mathbf{R}_{\mathrm{nm}}=\xi+\mathrm{na}+\mathrm{mb}
$$

then $A(\mathbf{K}) \exp (\mathbf{i K} \cdot \boldsymbol{\xi})$ is periodic with periods $\mathbf{A}$ and $\mathbf{B}$. Depending on $\boldsymbol{\xi}$, the array factor itself may have periods which are multiples of $\mathbf{A}$ and $\mathbf{B}$, or it may $\mathrm{b} \epsilon$ aperiodic. For instance, when $\boldsymbol{\xi}=\mathbf{b} / 2$ we have $\mathrm{A}(\mathbf{K}+\mathbf{B})=-\mathrm{A}(\mathbf{K})$ [see eq (29)] corresponding to a period of $2 \mathbf{B}$.

To gain the convenience of working with rectangular arrays we use the "doubled" lattice shown in figure 3. Because the lattice vectors

$$
\mathbf{a}^{\prime}=\frac{1}{2} \lambda \hat{\mathbf{x}}, \quad \mathbf{b}^{\prime}=\frac{3}{10} \lambda \hat{\mathrm{y}}
$$

are not primitive with respect to the centered rectangular sublattice, it is necessary to include virtual elements with 0 excitation at the locations

$$
\mathbf{R}_{\mathrm{nm}}^{\prime}=\mathrm{n} \mathbf{a}^{\prime}+\mathrm{m} \mathbf{b}^{\prime}
$$


when $\mathrm{n}+\mathrm{m}$ is even. (The prime is used to distinguish the doubled lattice from the centered rectangular lattice.)

One effect of using the nonprimitive basis, $\mathbf{a}^{\prime}$ and ' $\mathbf{b}^{\prime}$, is that the fundamental period is doubled in size as shown in figure 4. In addition to the periodicities

$$
\begin{gathered}
A\left(\mathbb{K} \pm \mathbf{A}^{\prime}\right)=A(\mathbb{K} \pm \mathbf{A})=A(\mathbb{K}), \quad A\left(\mathbb{K} \pm \mathbf{B}^{\prime}\right)=A(\mathbb{K}) \\
\mathbf{A}^{\prime}=\mathbf{A}=2 \mathrm{k} \hat{\mathbf{x}}, \quad \mathbf{B}^{\prime}=\frac{10}{3} \mathrm{k} \hat{\mathbf{y}}
\end{gathered}
$$

we have

$$
A(K \pm B)=-A(K)
$$

The minus sign in eq (29) occurs because the centered rectangular sublattice is offset from the origin. The fundamental period of the doubled lattice can be constructed from the fundamental period of the centered rectangular sublattice using eqs (27) and (29). The mapping is illustrated in figure 4.

\subsection{Transforming Between Array Factor and Excitation Matrix}

The array factor is given by

$$
A(\mathbf{K})=\sum_{\nu \mu} a_{\nu \mu}^{\prime} \exp \left(-\mathbf{i} \mathbf{K} \cdot \mathbf{R}_{\nu \mu}^{\prime}\right)
$$

where

$$
a_{n m}^{\prime}=a\left(R_{n m}^{\prime}\right)
$$

To produce the appropriate centered rectangular lattice, $a_{n m}^{\prime}$ must be 0 when $\mathrm{n}+\mathrm{m}$ is even $\left(\mathrm{a}_{00}^{\prime}=0\right)$.

For convenience in applying the FFT algorithm, we bias the summation indices so they will be positive:

$$
\mathrm{A}(\mathbf{K}) \exp \left(-\mathbf{i K} \cdot \mathbf{R}_{\mathrm{NM}}^{\prime}\right)=\sum_{\nu=0}^{\mathrm{P}-1} \sum_{\mu=0}^{\mathrm{Q}-1} \mathrm{a}_{\nu-\mathrm{N}, \mu-\mathrm{M}}^{\prime} \exp \left(-\mathbf{i} \mathbf{K} \cdot \mathbf{R}_{\nu \mu}^{\prime}\right)
$$


Here $N$ and $M$ have been chosen so that $a_{n m}^{\prime}=0$ when $n<-N$ or $m<-M ; P$ and $Q$ have been chosen so that $a_{n-N, m-M}^{\prime}=0$ when $n \geq P$ or $m \geq Q$. In effect, a new origin has been chosen so that the reference element coordinates are (N,M).

Let

$$
\mathrm{K}_{\mathrm{nm}}=\frac{\mathrm{n}}{\mathrm{P}} \frac{2 \pi}{\mathrm{a}^{\prime}} \hat{\mathbf{a}^{\prime}}+\frac{\mathrm{m}}{\mathrm{Q}} \frac{2 \pi}{\mathrm{b}^{\prime}} \hat{\mathbf{b}}^{\prime}=\frac{\mathrm{n}}{\mathrm{P}} \mathbf{A}^{\prime}+\frac{\mathrm{m}}{\mathrm{Q}} \mathbf{B}^{\prime}
$$

Then eq (30) may be rewritten

$$
\mathrm{A}\left(\mathrm{K}_{\mathrm{nm}}\right) \omega_{\mathrm{P}}^{-\mathrm{nN}} \omega_{\mathrm{Q}}^{-\mathrm{mM}}=\sum_{\nu=0}^{\mathrm{P}-1} \sum_{\mu=0}^{\mathrm{Q}-1} \mathrm{a}_{\nu-\mathrm{N}, \mu-\mathrm{M}}^{\prime} \omega_{\mathrm{P}}^{-\nu \mathrm{n}} \omega_{\mathrm{Q}}^{-\mu \mathrm{m}}
$$

where

$$
\omega_{\mathrm{P}} \equiv \exp \left(\frac{2 \pi i}{\mathrm{P}}\right), \quad \omega_{\mathrm{Q}} \equiv \exp \left(\frac{2 \pi i}{\mathrm{Q}}\right)
$$

If we define

$$
\begin{aligned}
& A\left(K_{n m}\right) \equiv F_{n+N, m+M} \omega_{P}^{n N} \omega_{Q}^{m M} \\
& a_{n m}^{\prime} \equiv f_{n+N, m+M} \omega_{P}^{-(n+N) N} \omega_{Q}^{-(m+M) M} \\
& -N \leq n \leq P-1-N, \quad-M \leq m \leq Q-1-M,
\end{aligned}
$$

then eq (32) becomes

$$
\begin{aligned}
& \mathrm{F}_{\mathrm{nm}}=\sum_{\nu=0}^{\mathrm{P}-1} \sum_{\mu=0}^{\mathrm{Q}-1} \mathrm{f}_{\nu \mu} \omega_{\mathrm{P}}^{-\nu \mathrm{n}} \omega_{\mathrm{Q}}^{-\mu \mathrm{m}} \\
& \mathrm{f}_{\mathrm{nm}}=\frac{1}{\mathrm{PQ}} \sum_{\nu=0}^{\mathrm{P}-1} \sum_{\mu=0}^{\mathrm{Q}-1} \mathrm{~F}_{\nu \mu} \omega_{\mathrm{P}}^{\nu \mathrm{n}} \omega_{\mathrm{Q}}^{\mu \mathrm{m}} .
\end{aligned}
$$

The sequences $F_{n m}$ and $f_{n m}$ are related by a two-dimensional DFT. The choice of phase factors in eqs (34) and (35) allows use of the natural range for the indices eq (36) without rearrangement of the outputs of the FFT routine.

The transformation between excitation matrix and array factor may be represented schematically as 


$$
\mathrm{a}^{\prime} \longleftrightarrow \begin{gathered}
\text { phase } \\
\text { factor }
\end{gathered} \mathrm{f} \longleftrightarrow \mathrm{FFT} \longleftrightarrow \mathrm{F} \longleftrightarrow \begin{array}{r}
\text { phase } \\
\text { factor }
\end{array} \mathrm{A}
$$

For the specific case used in the simulation, the excitation matrix (here denoted $\mathrm{c}_{\mathrm{nm}}$ ) is in a row and column format rather than in the cartesian format used in these notes. The relation to the broadside-beam excitation matrix $\alpha_{\mathrm{nm}}^{\prime}$ is

$$
\alpha_{\mathrm{nm}}^{\prime}=\mathrm{c}_{\mathrm{M}+\mathrm{I}-\mathrm{m}, \mathrm{N}+\mathrm{I}+\mathrm{n}}
$$

where

$$
\begin{aligned}
& \mathrm{N}=39, \quad \mathrm{M}=70 \\
& \mathrm{P} \geq 79, \quad \mathrm{Q} \geq 141 .
\end{aligned}
$$

\subsection{THE MERGED-SPECTRUM TECHNIQUE}

When the beam is steered to $\mathbf{K}_{0}$, the visible region of the form factor is a circle of radius $k$ centered on $-\mathbb{R}_{0}$ (see section 3.3 ). As figure 5 illustrates, when using only a broadside beam, extrapolation is necessary to estimate the form factor. (Coverage does not extend even to radius $k$ since the near-field scan area must be truncated.) From a practical point of view it is better to measure the array factor directly rather than to extrapolate. In the merged-spectrum technique, direct measurement is accomplished by steering the beam to make different regions of the form factor visible. Information from several beams is merged to give the fundamental period. Figure 6 shows an example (discussed below) using four beams.

\subsection{Choosing Parameters}

Three criteria must be considered:

(1) The density of points in $\mathbf{R}$ space must be chosen so that the requirement eq $(39 \mathrm{~b})$ is satisfied.

(2) The beams should be obtainable without quantization errors in the phase shifters.

(3) The beam directions must be chosen so that data points lie on a common grid in $\mathbf{K}$ space. 
To satisfy criterion ( 1 ) we take $P=128$ and $Q=256$, so the form factor is evaluated at the points

$$
\mathbf{K}_{\mathrm{nm}}=\frac{\mathrm{n}}{128} \mathbf{A}^{\prime}+\frac{\mathrm{m}}{256} \mathbf{B}^{\prime}
$$

With seven-bit phase shifters, the beam may be steered, without quantization error, to the directions $\mathbf{K}_{n m}$ when $\mathbf{m}$ is even. Thus, criteria (2) and (3) may be satisfied with the four beam directions

$$
\mathbf{K}_{ \pm 32, \pm 32}= \pm \frac{1}{4} \mathbf{A}^{\prime} \pm \frac{1}{8} \mathbf{B}^{\prime}=\left( \pm \frac{1}{2} \hat{\mathbf{x}} \pm \frac{5}{12} \hat{\mathrm{y}}\right) \mathrm{k}
$$

which are shown in figure 6 .

\subsection{Determining the Form Factor from Near-Field Data}

It is most convenient to collect near-field data so that the DFT will give the array coupling product $D^{a}(\mathbf{K})$ at appropriate $\mathbf{K}$ values without requiring additional interpolation. A comparison of eqs (6) and (31) yields

$$
\begin{aligned}
& N_{x} \Delta \mathrm{x}=0.5 \lambda P \\
& N_{y} \Delta y=0.3 \lambda Q .
\end{aligned}
$$

For example, let $P=128$ and $Q=256$ as in eq (40). The requirements eqs (5) and (42) can be satisfied with the choices $\Delta x=0.25 \lambda, \Delta y=0.3 \lambda$, and $\mathrm{N}_{\mathrm{x}}=\mathrm{N}_{\mathrm{y}}=256$. The dimensions of the measurement plane are $64 \lambda$ by $77 \lambda$, whereas the array is roughly $42 \lambda$ in diameter. To determine $A_{0}(K)$ over a fundamental period, $\mathrm{D}^{\mathrm{a}}(\mathbf{K})$ must be known [for each steering angle eq (41)] over the central rectangle of figure $6\left(\theta \leq 40.6^{\circ}\right)$. According to the truncation criterion of section 2.4, calculated results should be valid in this region if the spacing between scan plane and array is less than about $14 \lambda$.

Near-field measurements are transformed in the standard manner (FFT) to obtain $D^{a}\left(\mathbf{K}_{\mathrm{nm}}\right)$. From eqs (3) and (18) we have

$$
A_{0}\left(\mathbf{K}_{n m}-\mathbf{K}_{0}\right)=D^{a}\left(\mathbf{K}_{n m}\right) / D^{e}\left(\mathbf{K}_{n m}\right)
$$

where $D^{e}\left(K_{n m}\right)$ is the coupling product between the probe and an "average element." $D^{e}\left(\mathbf{K}_{n m}\right)$ is determined independently, and it must be known at least 
for the central rectangular region indicated in figure 6 . For each beam direction eq (43) determines $A_{0}(K)$ in a disk of radius $K \leq k$ about the point $\mathbf{K}=-\mathbf{K}_{0}$.

\subsection{Combining Beams}

One way to combine the spectral information from the beams used in figure 6 is to simply let each beam determine $A_{0}(\mathbf{K})$ in the corresponding quadrant, perhaps averaging along the common boundaries. An alternative method would be to construct a weighted average in the overlap regions. The weights would be chosen proportional to some experimental measure of confidence. The second method allows more flexibility in choosing the location and number of beams in the merge.

Once the form factor has been determined on the fundamental period of the centered rectangular sublattice, the data can be extended over the fundamental period of the doubled lattice as shown in figure 4. Finally, the results of section 3.5 can be applied to transform from the form factor to the excitation $\operatorname{matrix} \alpha_{n m}^{\prime}$.

\subsection{SIMULATION FLOW}

The computer simulation, outlined in the flow charts of figures 7 and 8 , implements the theory of the preceding sections and provides a means for testing various aspects of the spectral merge process. Figure 7 illustrates the steps in obtaining the simulated near-field data which will be used for the merged-spectrum analysis. The procedure of figure 8 uses the near-field data for each of the steered beams to calculate the broadside-beam excitation matrix from the merged spectrum. Implementation of the blocks in figure 8 will be completed in the next phase of the study. The final result depicted in figure 8 should be the array excitation matrix input initially into the simulation process of figure 7. Any differences are due to measurement errors and effects of the merged-spectrum technique. Through simulation, we can determine the most critical errors and the limits of accuracy.

\subsection{Array Mode1}

The array used in the simulation is shown schematically in figure 9. The 4350 active elements are arranged in 79 columns and 141 rows (in a nearly 
circular region) on a centered rectangular lattice as shown in figures 1 and 9. To gain the convenience of working with a rectangular lattice, we add a virtual element between each actual element along the columns as shown in figures 3 and 10. Since virtual elements are assigned 0 amplitude in the excitation matrix, the performance of the array is not altered. Nominal element spacings in this doubled array are $a^{\prime}=0.5 \lambda$ in the $x$ direction (between columns) and $b^{\prime}=0.3 \lambda$ in the $y$ direction (between rows), resulting in an aperture approximately $42 \lambda$ in diameter; however, spacing can be changed, as desired. The array is further extended with virtual elements (zero filling) so that it is rectangular in shape with $P \geq 79$ columns and $Q \geq 141$ rows. Efficient use of the FFT dictates that $P$ and $Q$ should be powers of 2; for example, $\mathrm{P}=128, \mathrm{Q}=256$.

\subsection{Simulation Examples}

The first simulation example is for a broadside beam with no errors in any of the elements. Figures 11 through 14 are center line $(x=0$ and $y=0$ ) plots of the excitation matrix along with the corresponding field amplitude on measurement planes at $20 \mathrm{~cm}$ and $50 \mathrm{~cm}$. The field amplitudes, which are normalized to $0 \mathrm{~dB}$ peak, were calculated using eq (13). (The frequency is $3 \mathrm{GHz}$ for all computations.)

The broadside-beam array (form) factor was calculated using eq (30) with $\mathrm{P}=\mathrm{Q}=512$ and results are shown in figures 15 through 18 . Figures 15 and 16 are plots of the array factor over the fundamental period of the doubled lattice: $\quad-1<k_{x} / k \leq 1,-5 / 3<k_{y} / k \leq 5 / 3$. These figures confirm that the fundamental period for the doubled array can be determined if the array factor is known in the subregion (shaded in fig. 4) which corresponds to the fundamental period of the actual array without virtual elements. (See the discussion of fig. 4 in section 3.3.) Thus "doubling" of the array, which is done for computational convenience, does not cost anything from a measurement point of view. Figures 17 and 18 show the array factor in the principal planes. [The horizontal bands in the array factor evident in figure 15 apparently arise because the elements are driven pairwise in the $y$ direction (fig. 12). Similar vertical bands might be expected in figure 15, as well, except that the $\mathrm{y}=0$ row (the excitations are plotted in fig. 11) is the only row with elements driven in pairs.]

The next step in the simulation is the calculation of the measured data on a specified measurement plane. The array factor is first multiplied by the 
coupling product between probe and average element patterns and then by the factor $\exp \left(i \gamma z_{0}\right)$ before transforming to the near-field with the FFT [see eq (13)]. A typical spectrum for both the probe and element is shown in figures 19 and 20 with the amplitude of the coupling product $D^{e}(\hat{\mathbf{k}})$ shown in figure 21. Since both the probe and element have very broad patterns, they have little effect on the spectrum of the low sidelobe array. (The major effect occurs when $\mathrm{K} / \mathrm{k}>0.6$ where the coupling product is reduced by 10 to 30 $\mathrm{dB}$ due to the combined effect of probe and element patterns.) The factor $\exp \left(i \gamma z_{0}\right)$ is important, however. Within the visible region (fig. 22), where $\gamma$ is real, it reflects the phase progression of plane waves propagating from the aperture to the measurement plane. Beyond the visible region (fig. 23), where $\gamma$ is imaginary, it reflects the exponential attenuation of the evanescent components of the spectrum.

Figures 24 through 27 represent error-free data from an open-ended waveguide probe on measurement planes $20 \mathrm{~cm}$ and $50 \mathrm{~cm}$ from the aperture of an array with no faulty or misaligned elements. The program provides the option of introducing various errors and example array factors are shown in figures 28 through 31. Here, the excitation phases have been modified, by adding a sinusoidal error in the $\mathrm{x}$ and/or $\mathrm{y}$ directions, to produce paired error lobes in addition to the main beam. Other errors will be simulated in future studies.

In addition to the broadside beams illustrated thus far, steered beams can be produced by imposing a linearly varying phase on the excitation matrix (section 3.2). An example array factor is shown in figure 32. The array factor for a steered beam can be obtained by translating the form factor (fig. 15) as indicated by eq (17). Multiple beam directions are required for the merged-spectrum simulation.

\subsection{SUMMARY}

Computer code and supporting analysis have been developed as the basis for a thorough evaluation by simulation of the merged-spectrum technique for aligning planar arrays. The actual evaluation of the merged-spectrum technique will be described in a future report. 


\section{REFERENCES}

[1] Patton, W.T. Phased array alignment with planar near-field scanning; or determining element excitation from planar near-field data. In Proc. Antenna Appl. Symp., Univ. Illinois; Sept. 1981.

[2] Kerns, D.M. Plane-wave scattering-matrix theory of antennas and antennaantenna interactions. Nat. Bur. Stand. (U.S.) Monograph 162; June 1981.

[3] Ma, M.T. Theory and application of antenna arrays. New York: Wiley; 1974. 


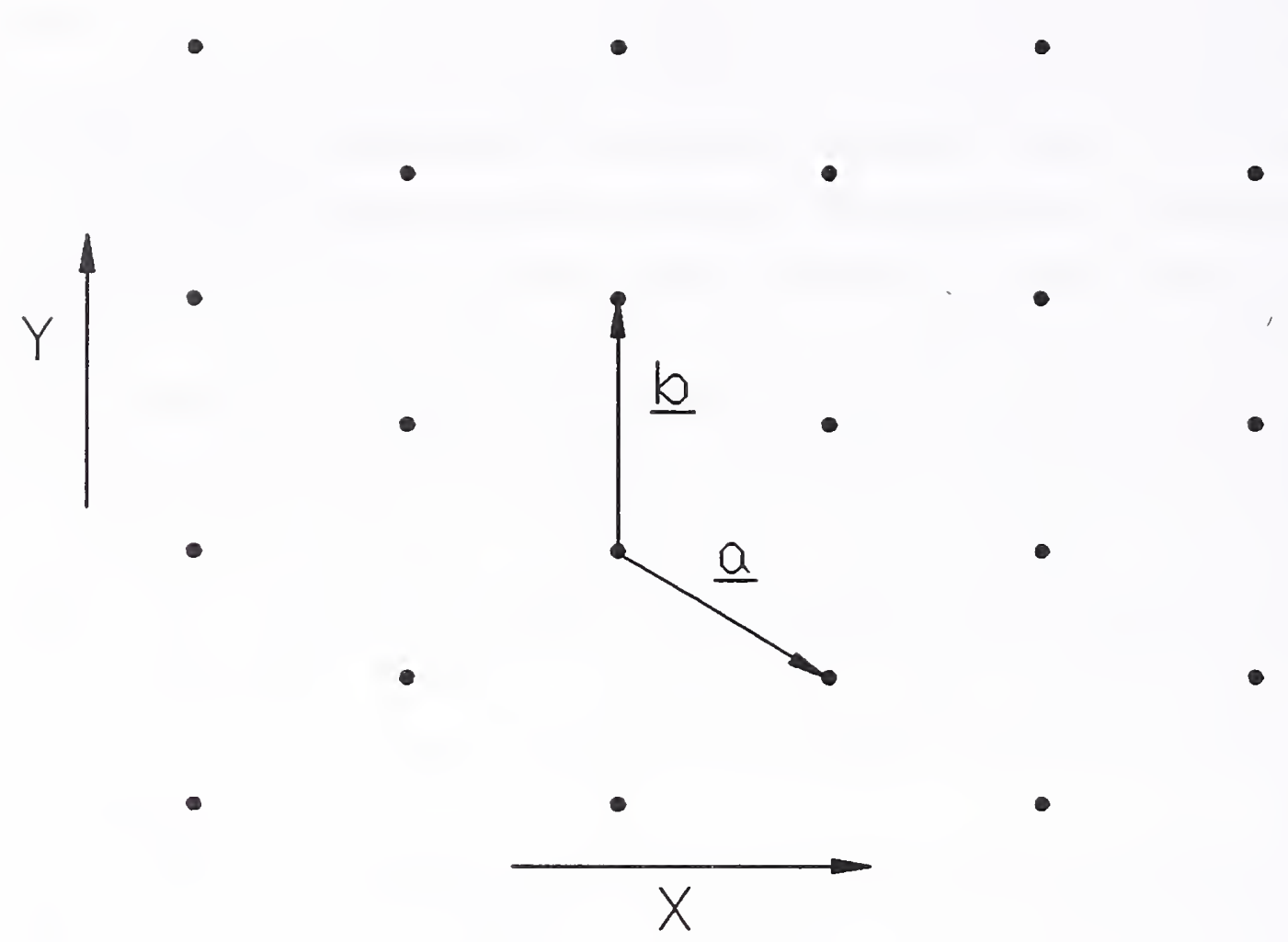

Figure 1. A centered rectangular lattice showing a set of fundamental (and primitive) lattice vectors. $a=\lambda(1 / 2 \hat{x}-3 / 10 \hat{y}), b=\lambda 3 / 5 \hat{y}$.

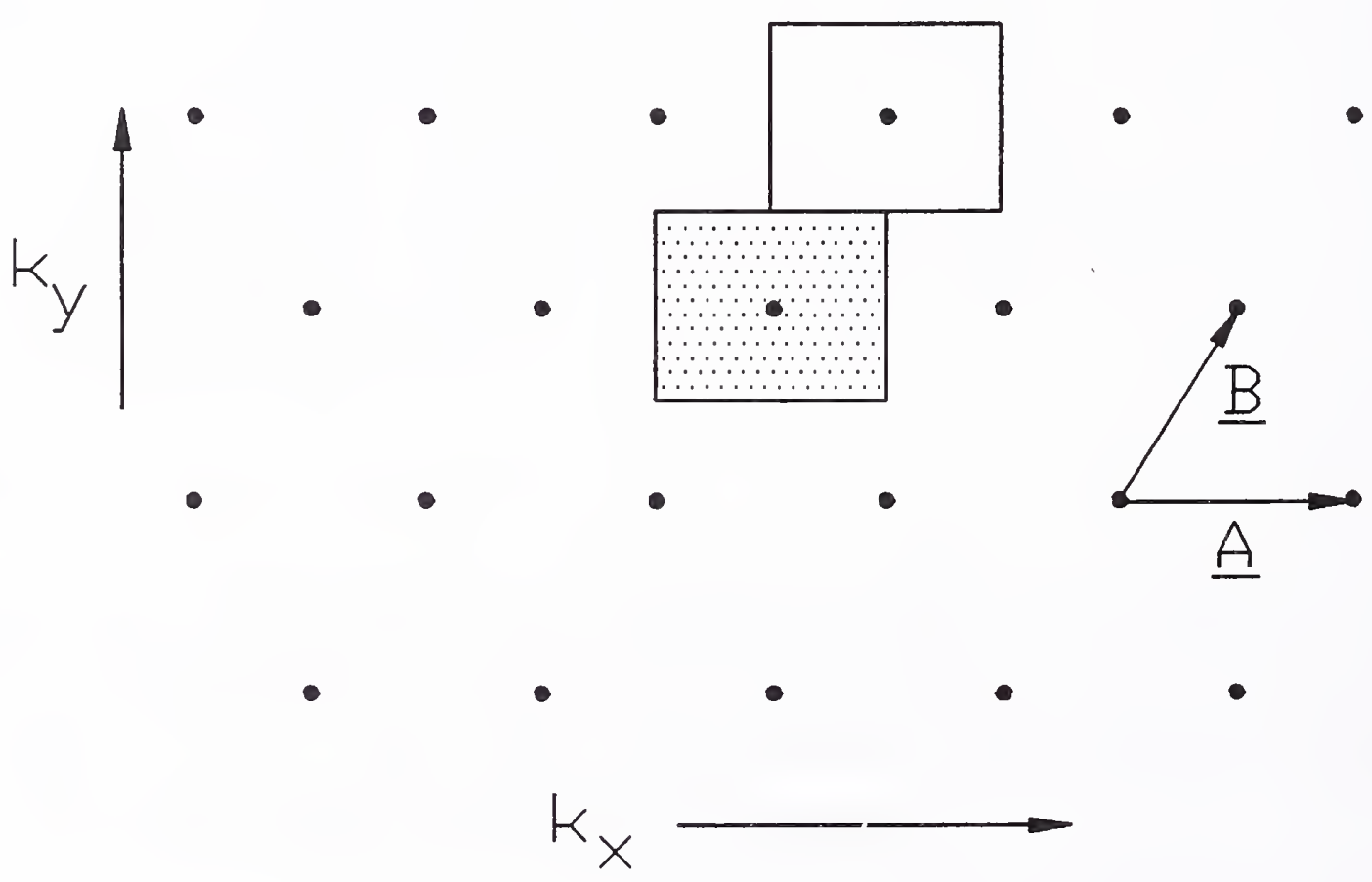

Figure 2. The reciprocal lattice for the lattice of figure $1 . A=2 k \hat{x}$, $B=k(\hat{x}+5 / 3 \hat{y})$. If the array factor is known on the fundamental period (shaded), then it may be determined everywhere by periodic repetition. For example, in the open rectangle $A(K)=A(K-B)$. 


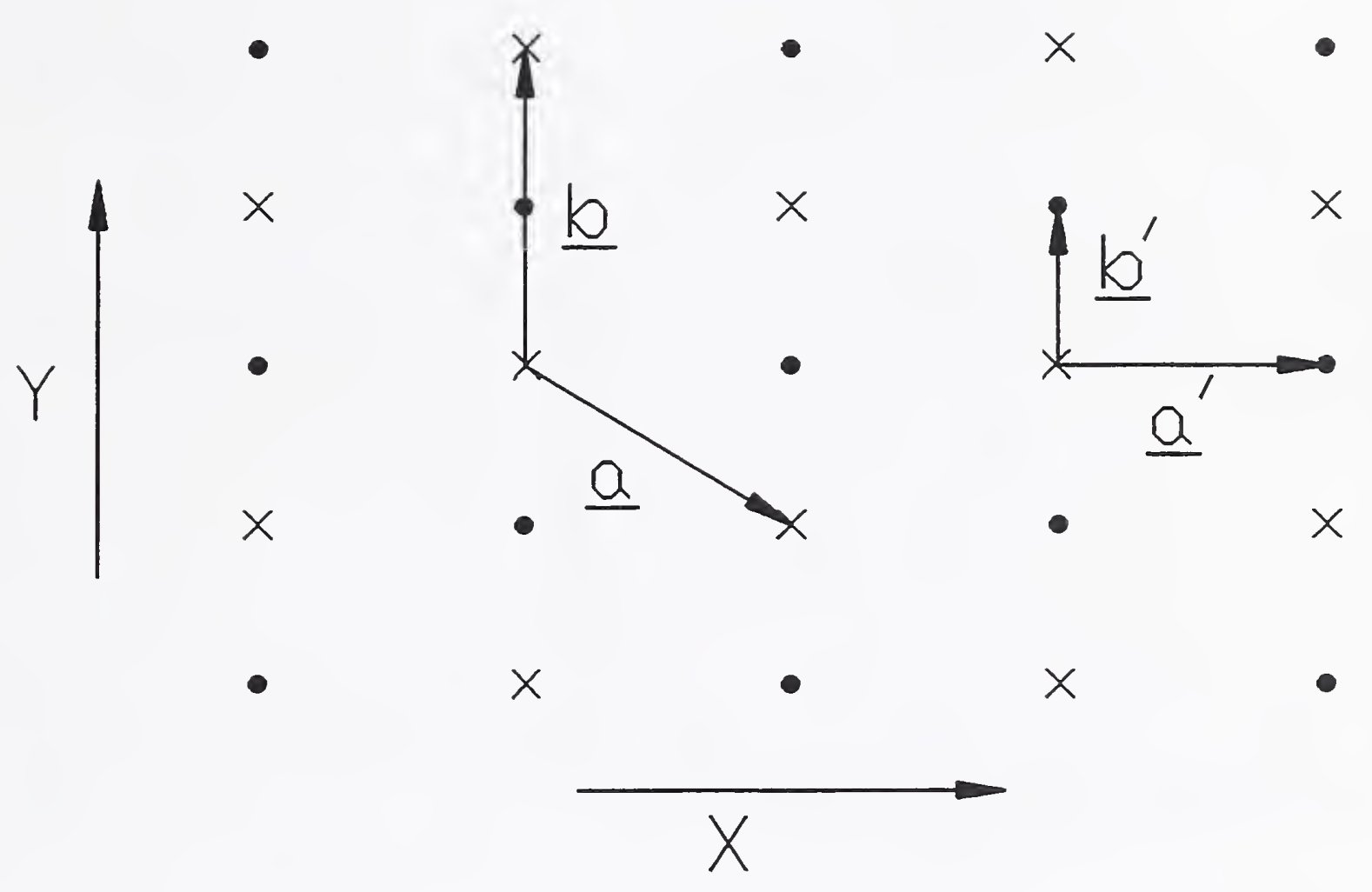

Figure 3. A rectangular lattice obtained from the centered rectangular lattice of figure 1 by adding interstitial points. $a^{\prime}=\lambda 1 / 2 \hat{x}$, $\mathrm{b}^{\prime}=\lambda 3 / 10 \hat{\mathrm{y}}$. Element positions are marked with $\mathrm{x}^{\prime} \mathrm{s} . \quad\left(\alpha^{\prime}{ }_{\mathrm{nm}}=0, \mathrm{n}+\mathrm{m}\right.$ even.)

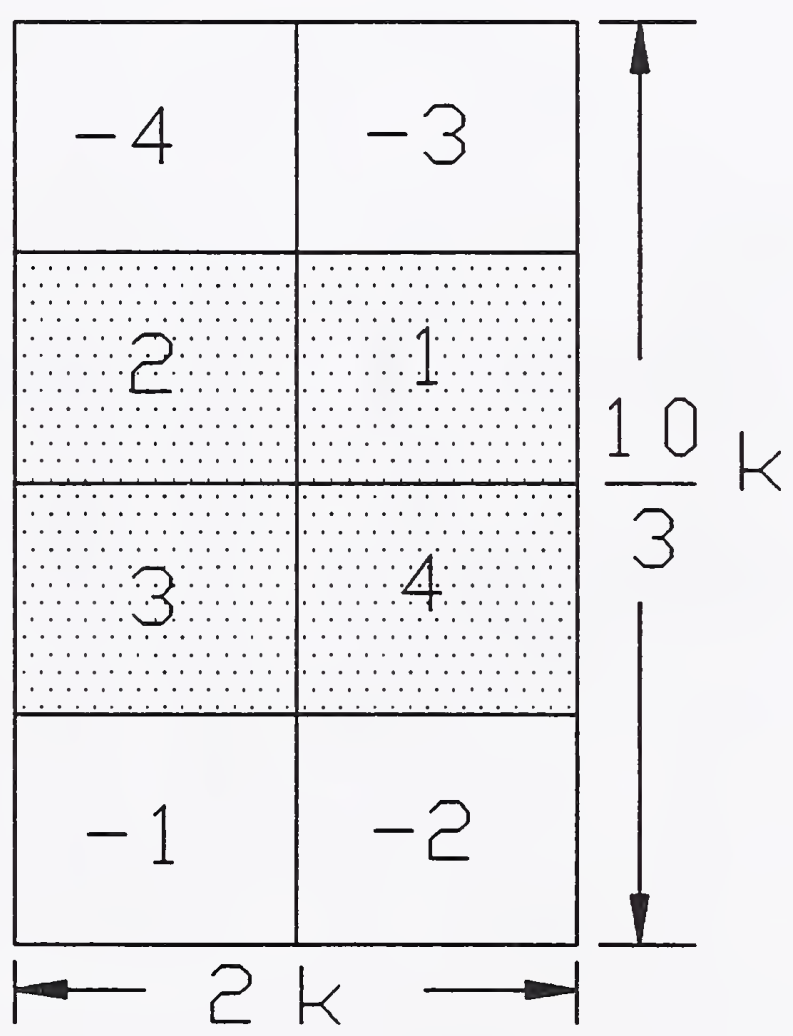

Figure 4. A fundamental period in the reciprocal lattice for the doubled lattice of figure 3. $A(K)$ may be determined everywhere from its values on the fundamental period of the centered rectangular sublattice (shaded); The mapping is indicated by quadrant. The minus signs occur because the centered rectangular sublattice is offset from the origin. 


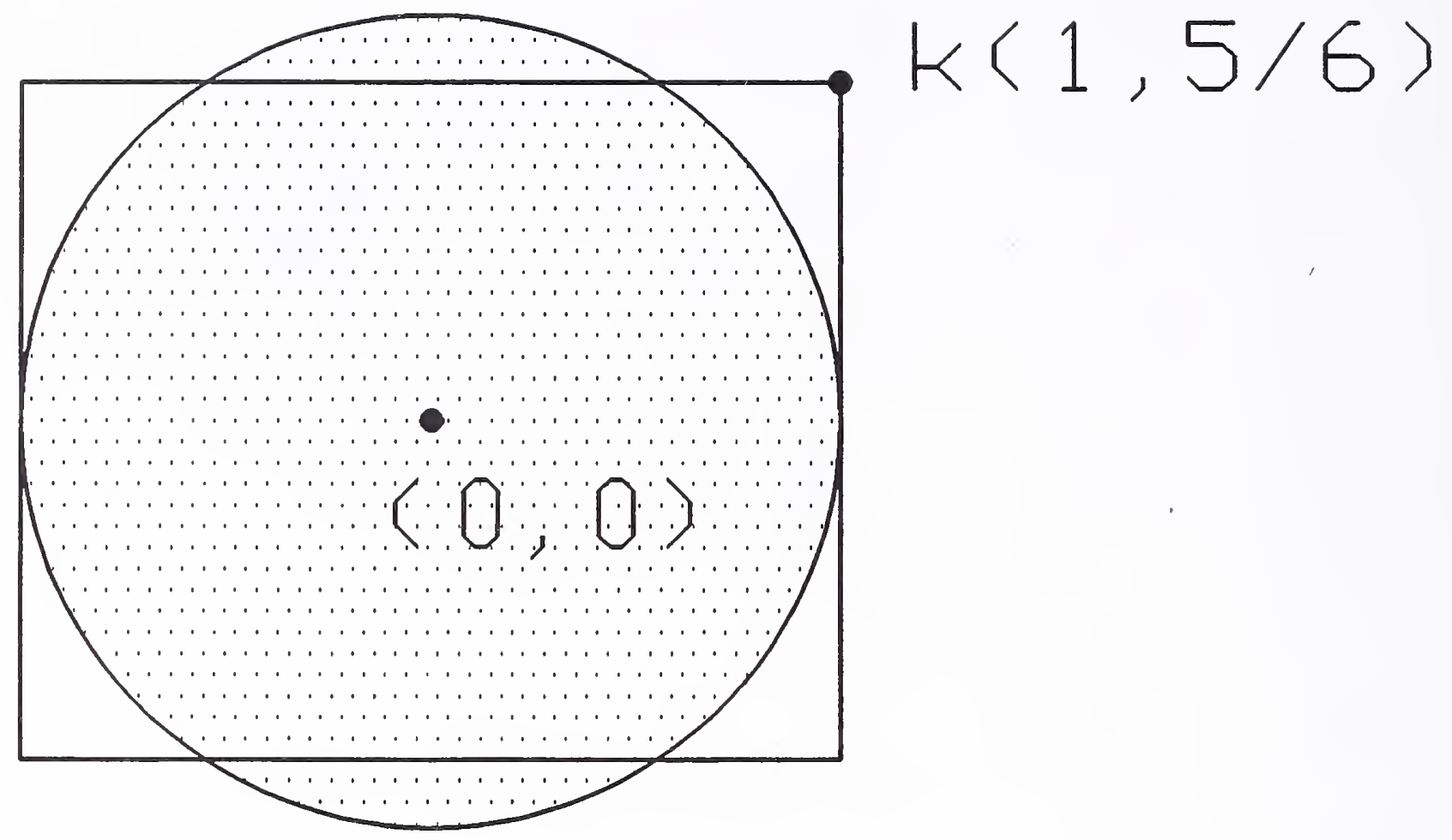

Figure 5. The visible region for a broadside beam is the shaded disk. Extrapolation would be necessary to determine the form factor from this information. In the merged-spectrum technique, direct measurement of the fundamental period (rectangle) is accomplished via beam steering.

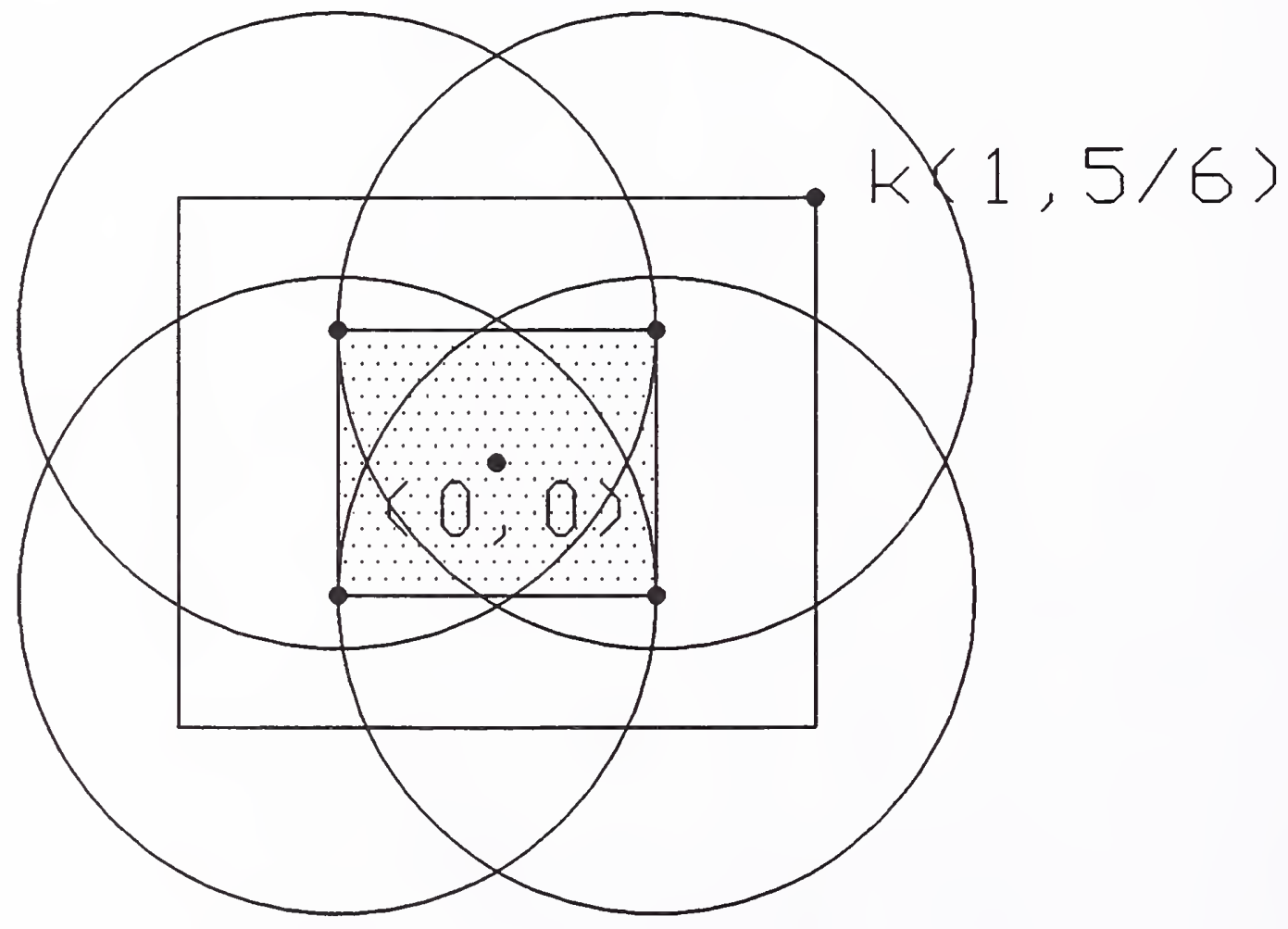

Figure 6 . When the beam is steered to the four locations

$\mathrm{k}_{0}=\mathrm{k}( \pm 1 / 2 \hat{\mathrm{x}} \pm 5 / 12 \hat{\mathrm{y}})$ marked with $\bullet ' s$, the entire fundamental period can be measured directly. The visible region corresponding to each beam direction is indicated by a circle. The shaded rectangle $\left(\theta \leq 40.60^{\circ}\right)$ is the smallest region in which coupling products must be determined. 


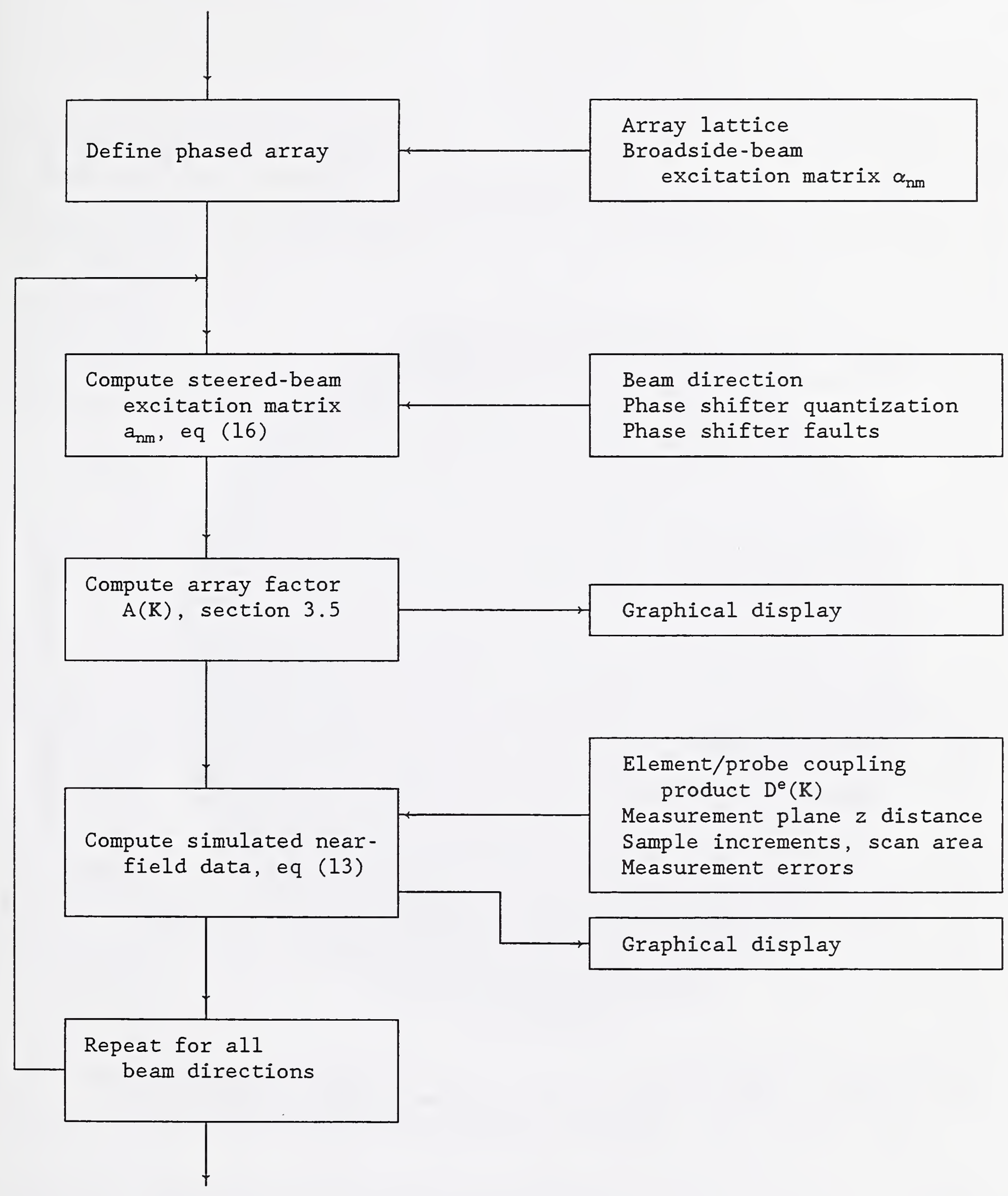

Figure 7. Block diagram showing the phased-array simulation and the nearfield measurement simulation. 


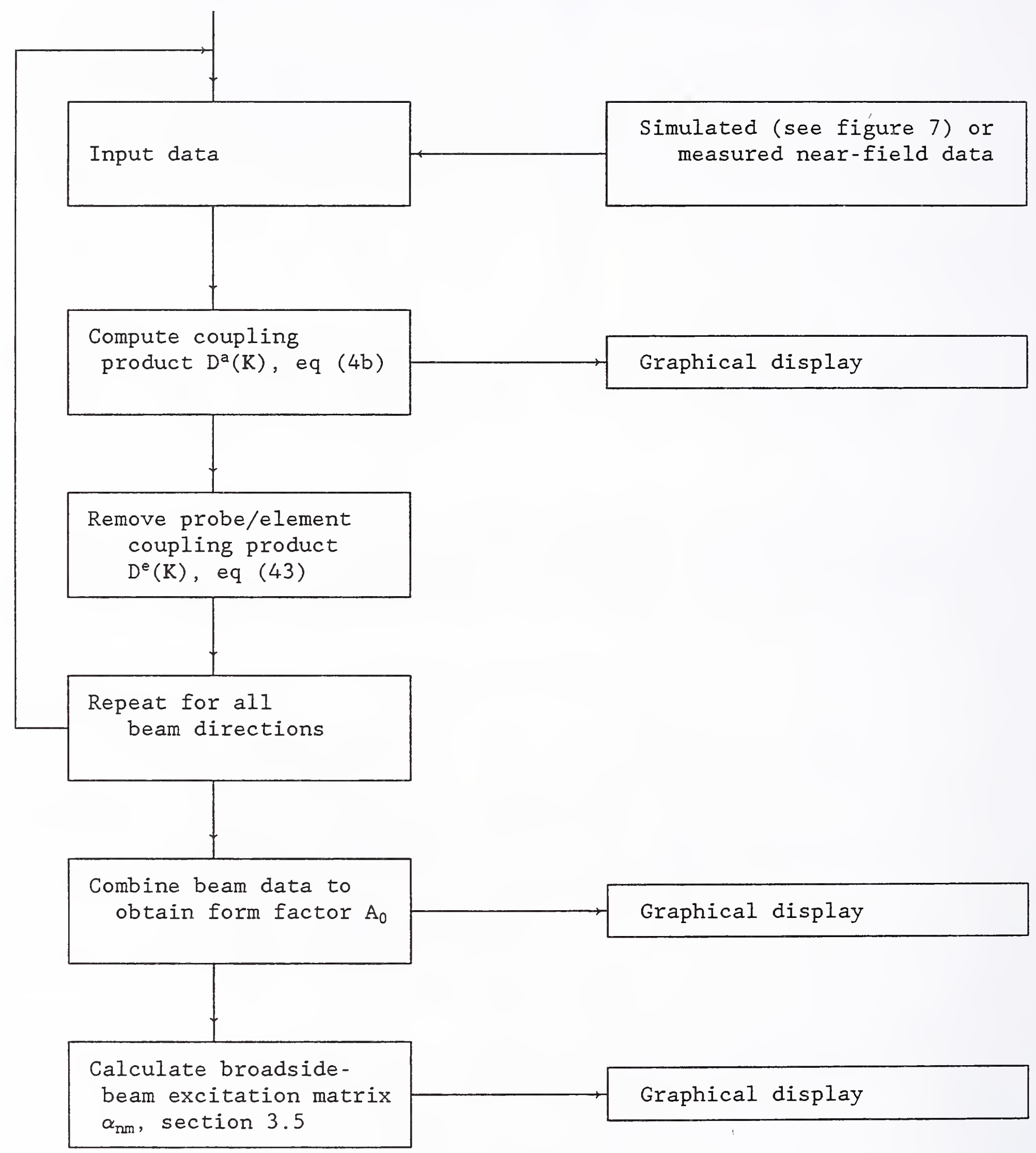

Figure 8. Block diagram showing the merged-spectrum technique for determining the phased-array excitation matrix. 


\section{ACTUAL ELEMENTS IN ARRAY}

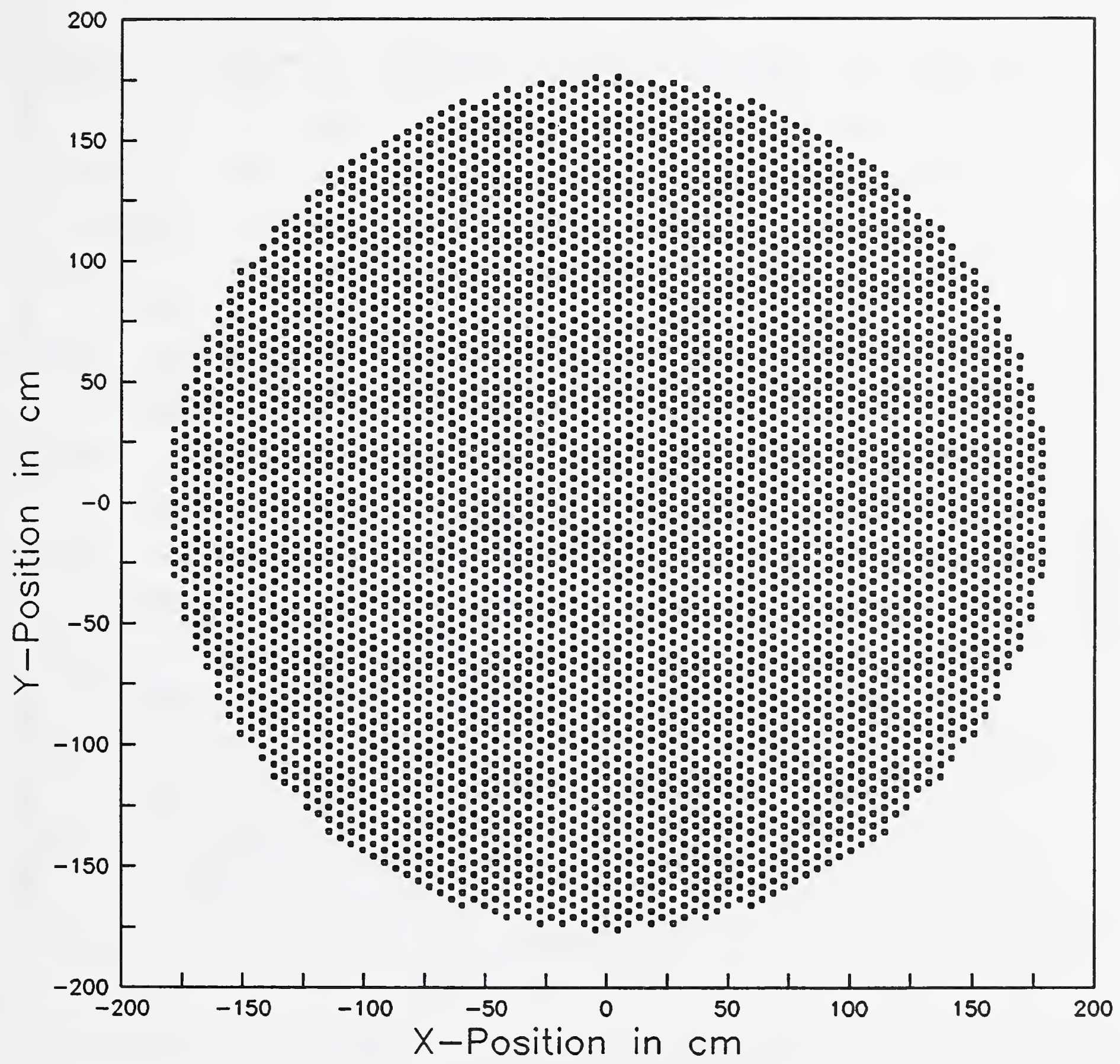

Figure 9. Schematic of the phased array showing location of active elements. 


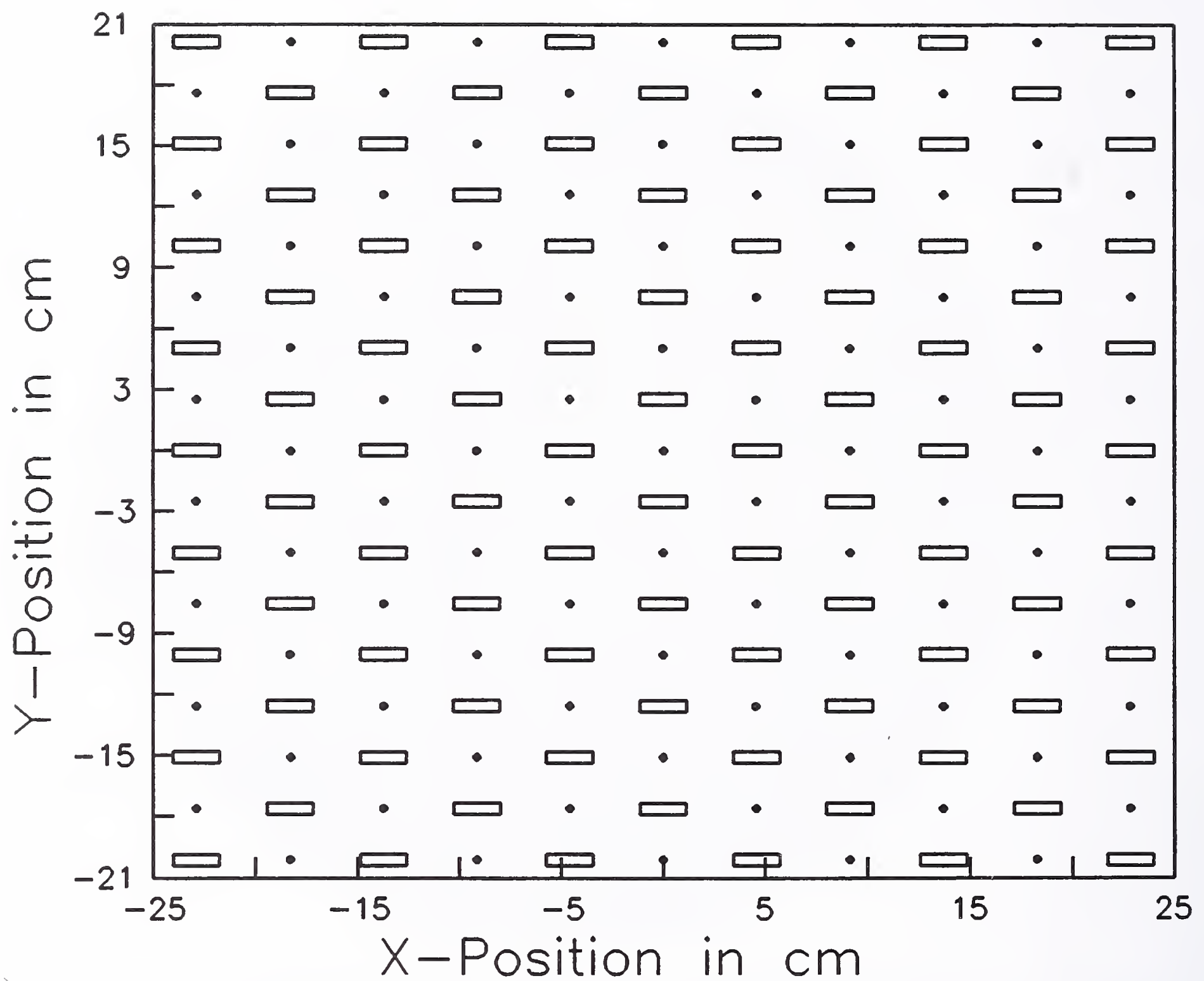

Figure 10. Schematic of the central part of the phased array showing the location of active elements $(\square)$ and virtual elements $(\bullet)$. 
$Z$ distance $20 \mathrm{~cm}$

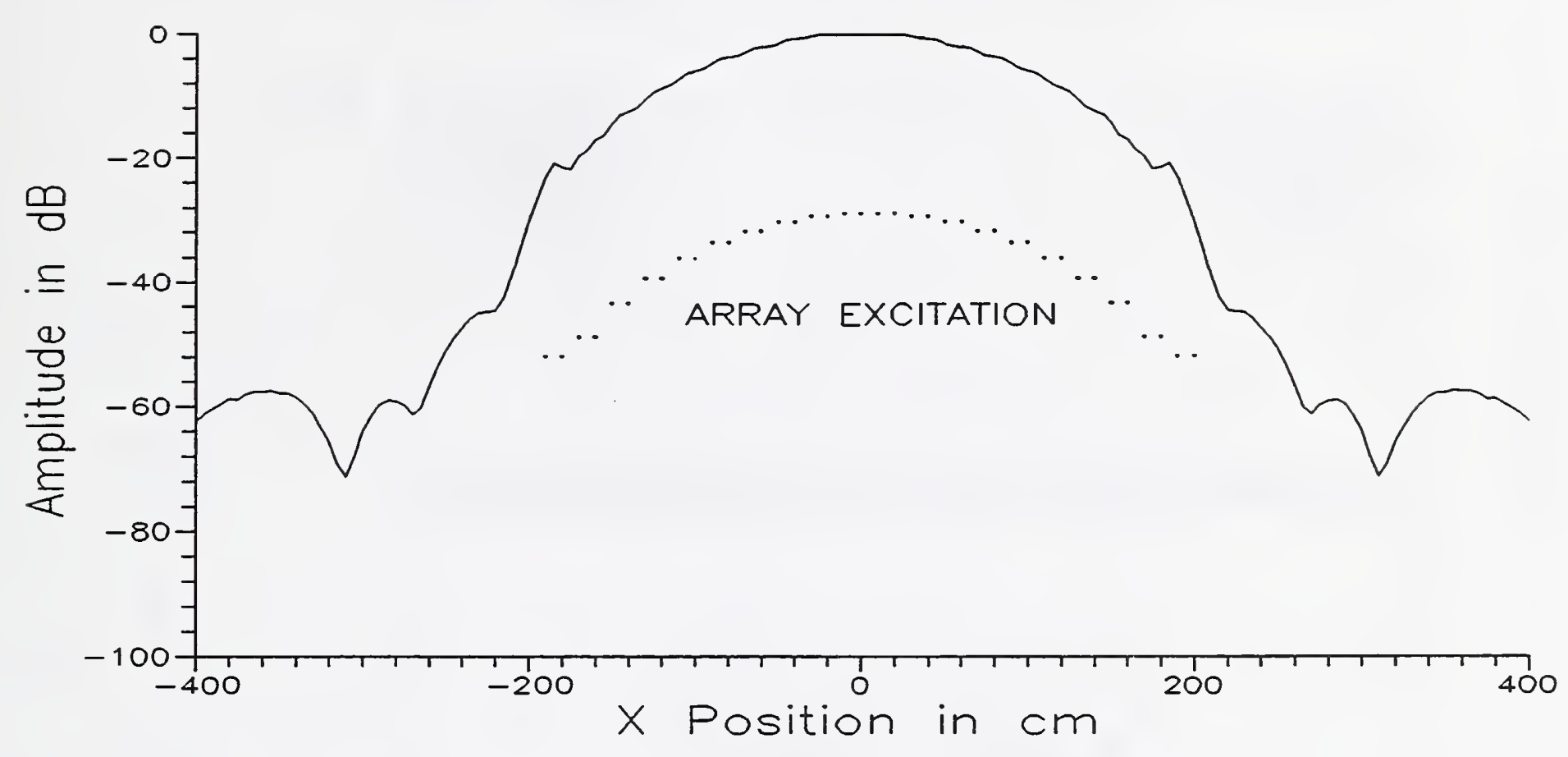

Figure 11. Relative amplitude excitations (dots) of elements along the center row, $y=0$, and field amplitude (solid line), normalized to 0 dB peak, at $z=20 \mathrm{~cm}$. Frequency is $3 \mathrm{GHz}$.

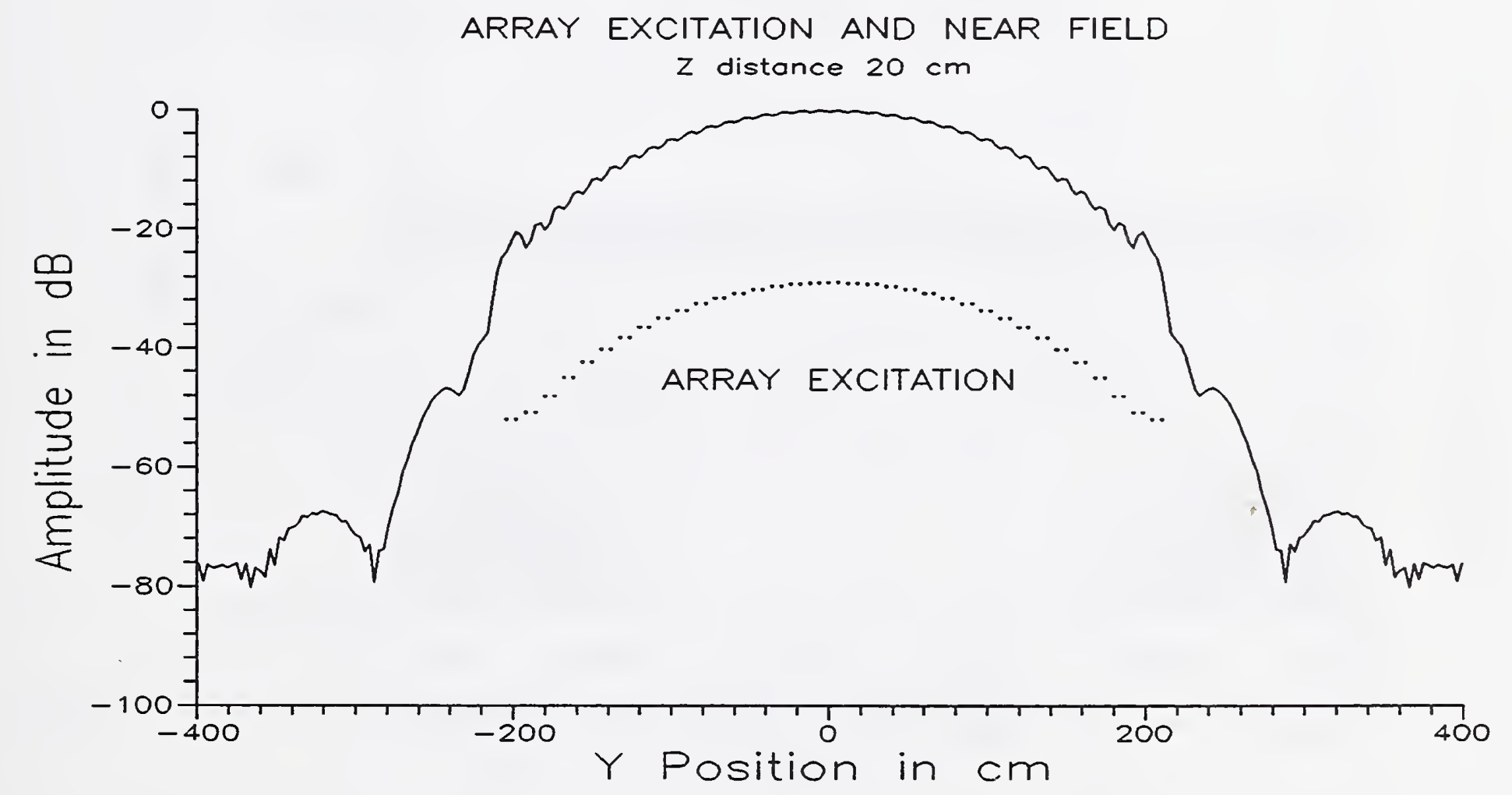

Figure 12. Relative amplitude excitations (dots) of elements along the center column, $\mathrm{x}=0$, and field amplitude (solid line), normalized to $0 \mathrm{~dB}$ peak, at $z=20 \mathrm{~cm}$. Frequency is $3 \mathrm{GHz}$. 


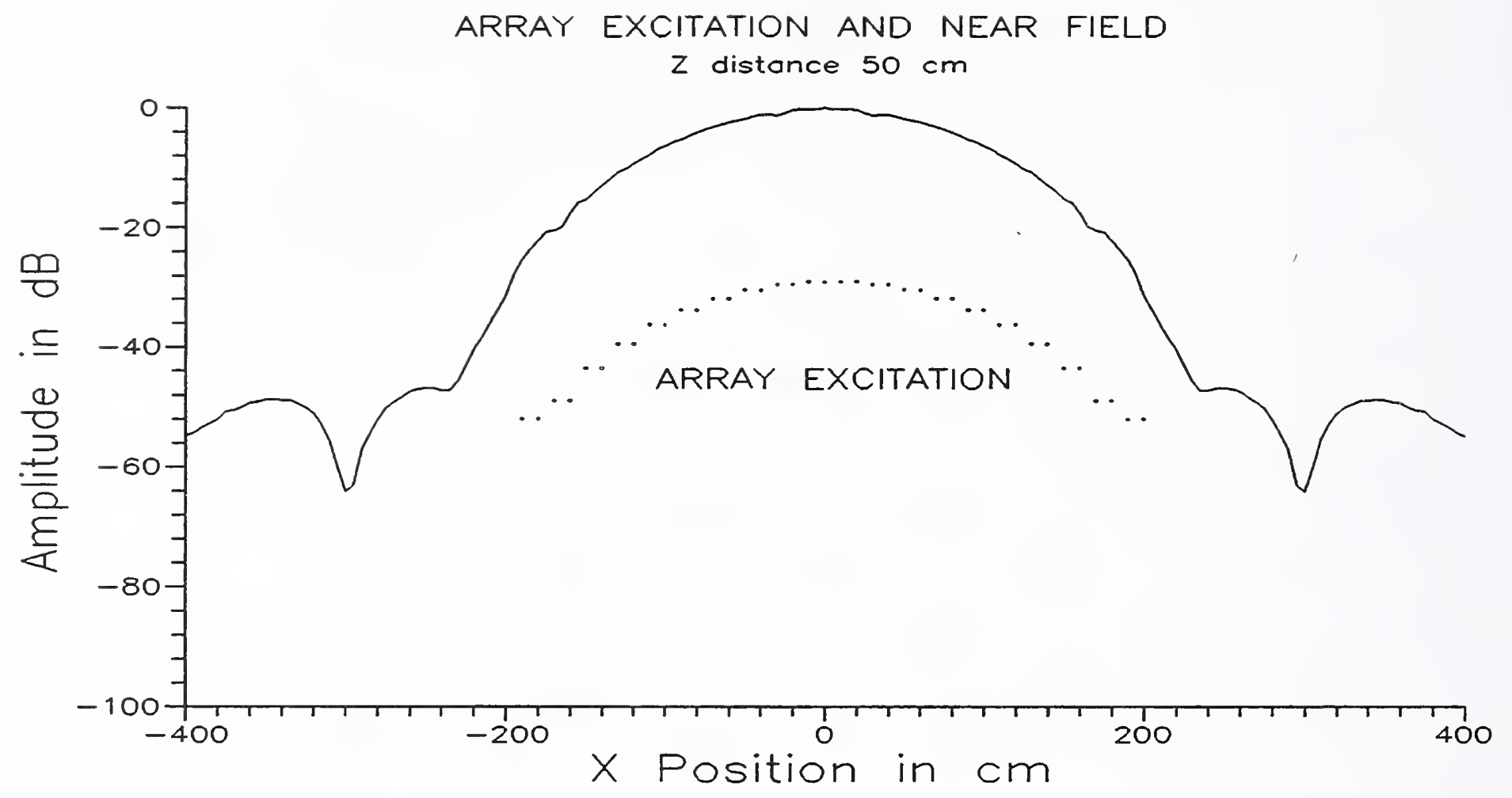

Figure 13. Relative amplitude excitations (dots) of elements along the center row, $\mathrm{y}=0$, and field amplitude (solid line), normalized to $0 \mathrm{~dB}$ peak, at $z=50 \mathrm{~cm}$. Frequency is $3 \mathrm{GHz}$.

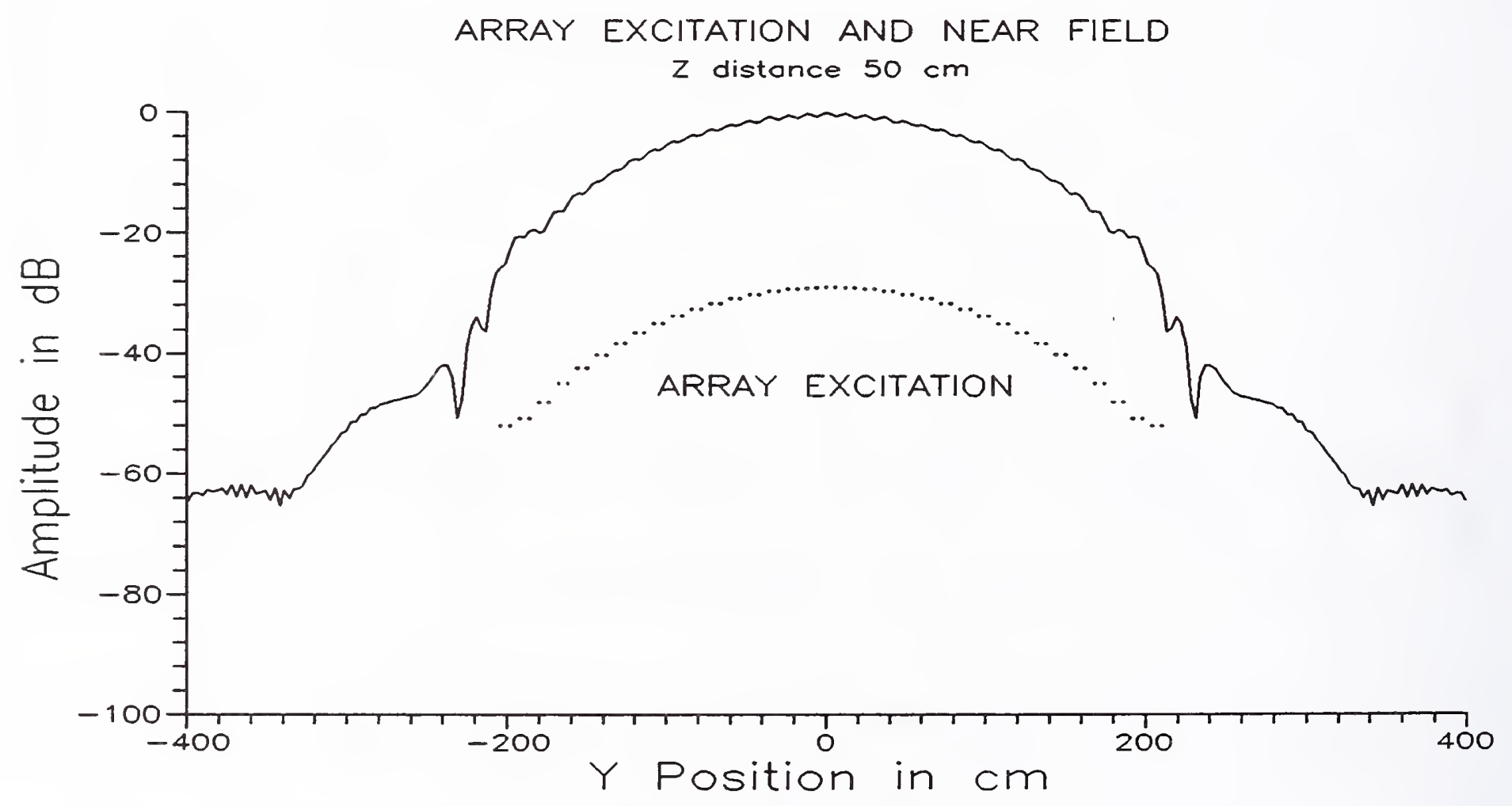

Figure 14. Relative amplitude excitations (dots) of elements along the center column, $x=0$, and field amplitude (solid line), normalized to $0 \mathrm{~dB}$ peak, at $z=50 \mathrm{~cm}$. Frequency is $3 \mathrm{GHz}$. 


\section{$10 \mathrm{~dB}$ Contours $(0,-60)$}

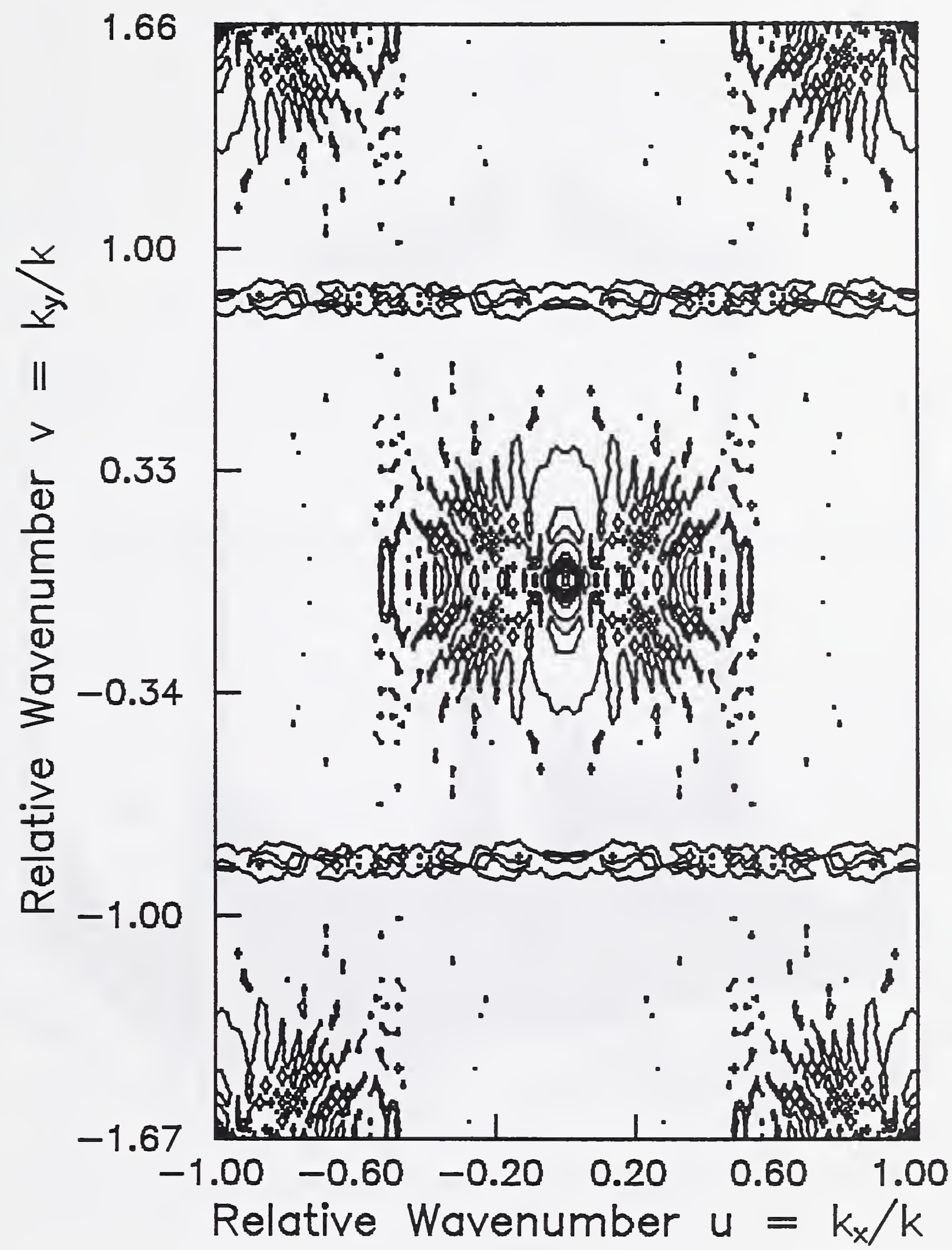

Figure 15. Contour plot of the array factor for the doubled array composed of active and virtual elements. 


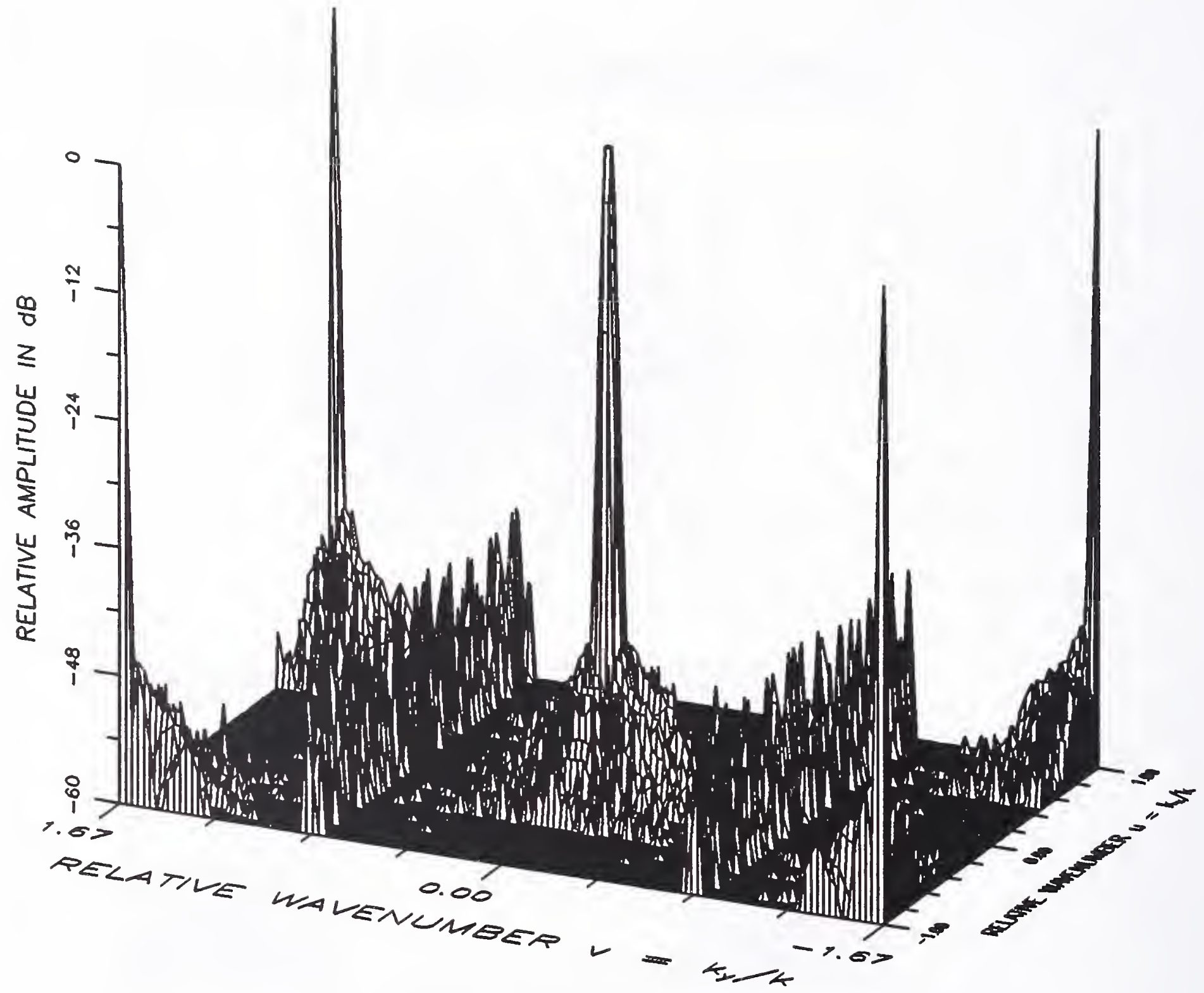

Figure 16. Perspective plot of the array factor for the doubled array composed of active and virtual elements. 


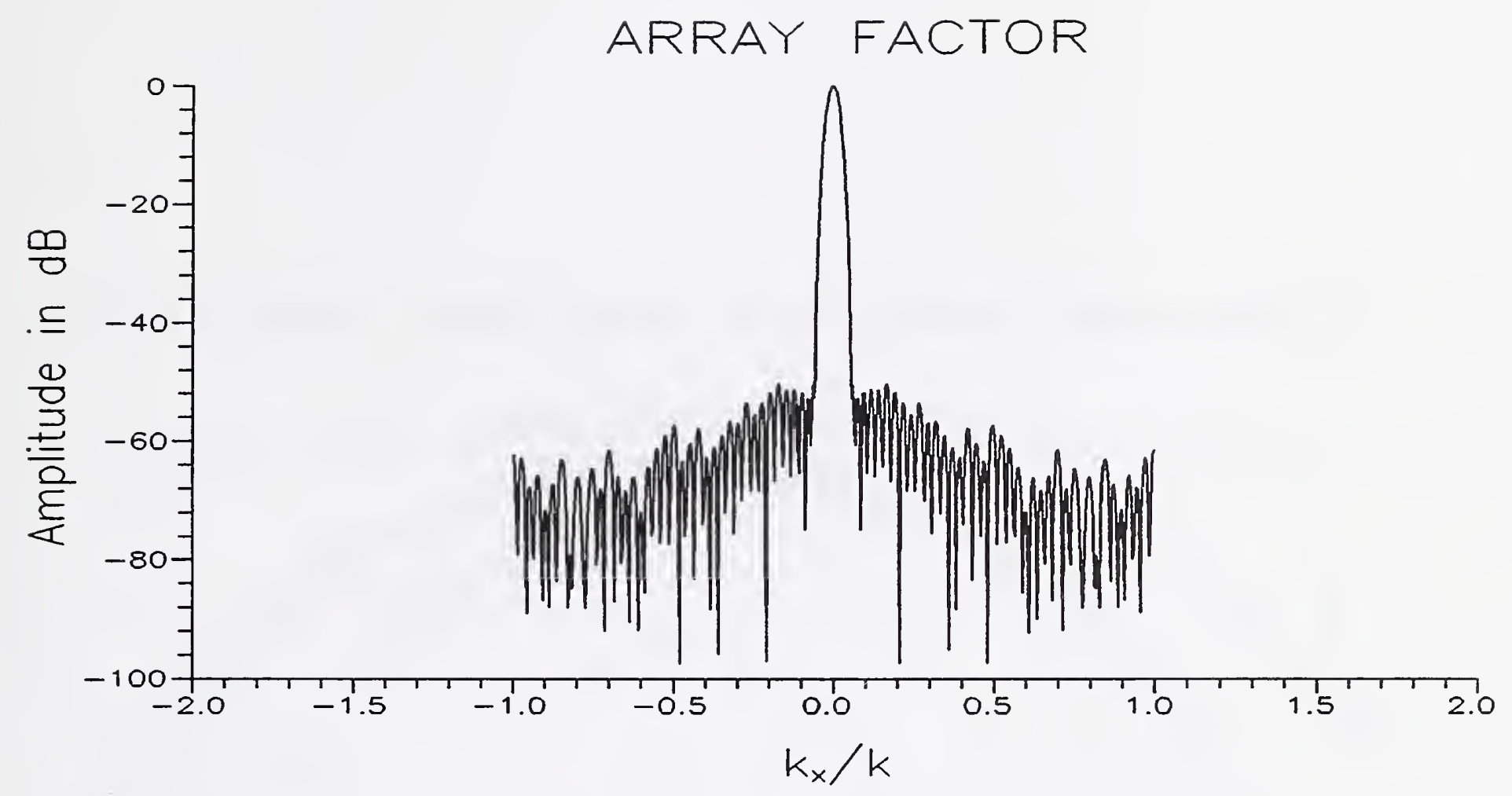

Figure 17. Principal plane $\left(k_{y}=0\right)$ cut of the array factor for the doubled array composed of active and virtual elements.

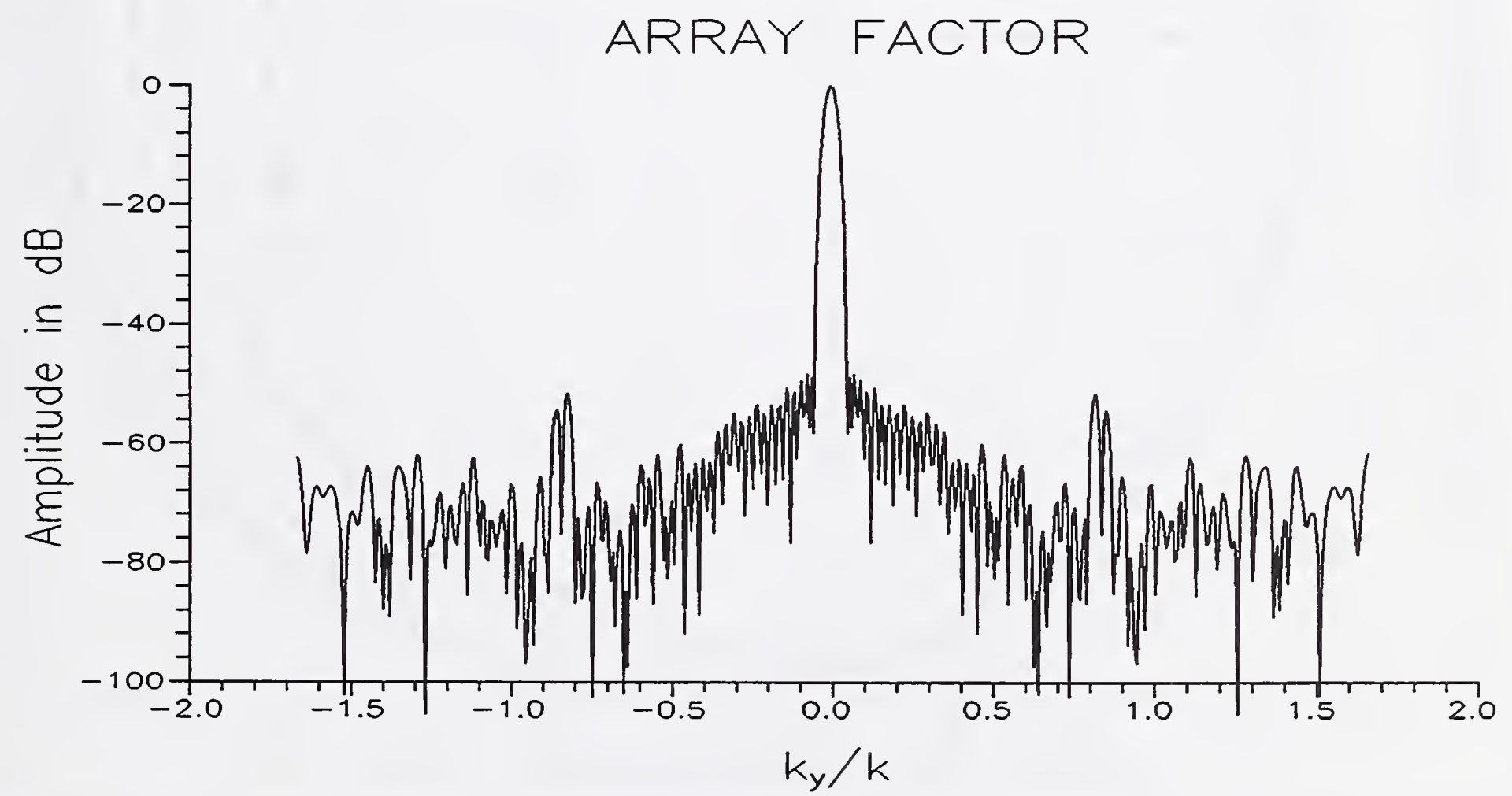

Figure 18. Principal plane $\left(k_{x}=0\right)$ cut of the array factor for the doubled array composed of active and virtual elements. 
OPEN-END WAVEGUIDE SPECTRUM, AMPLITUDE

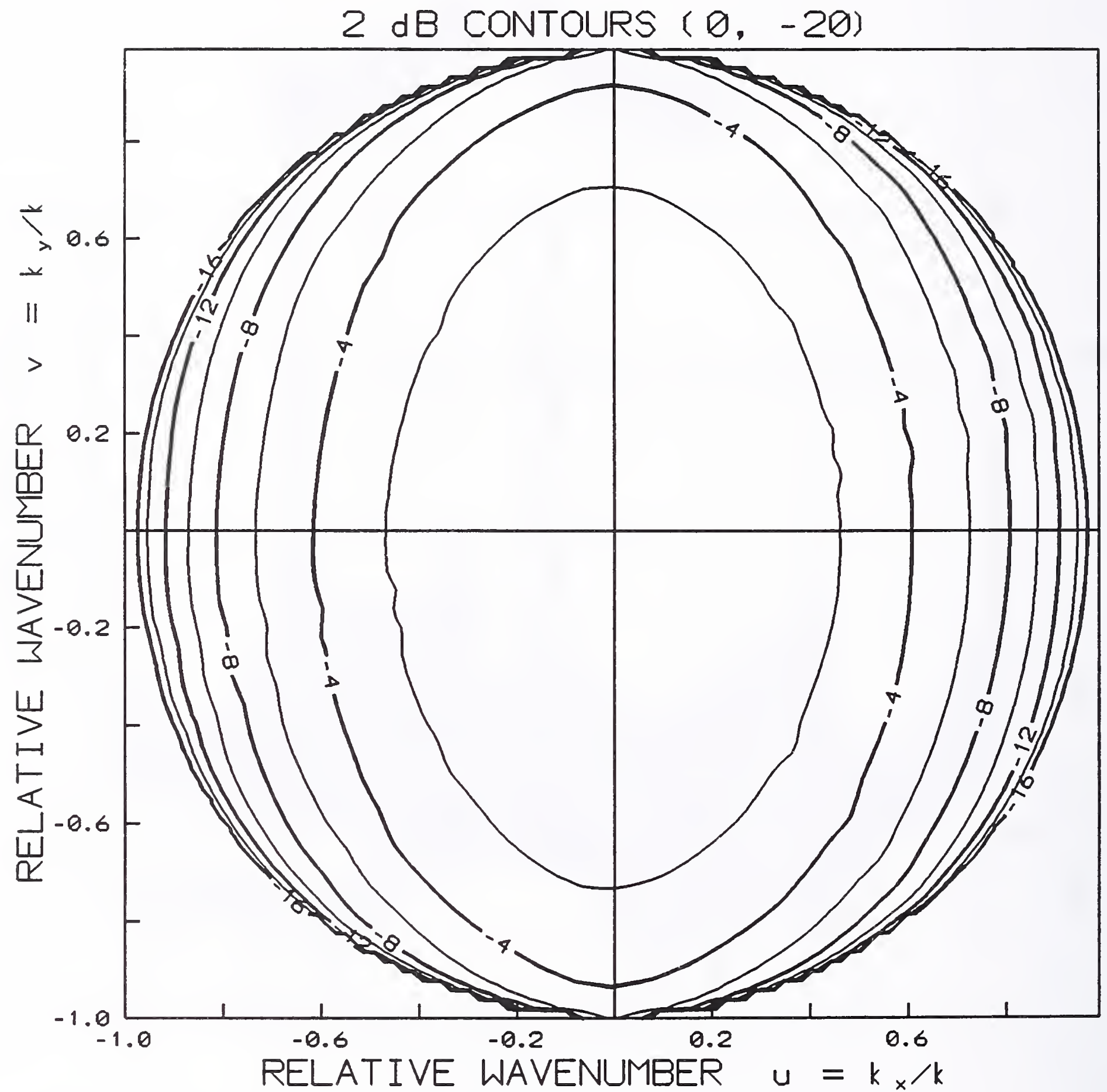

Figure 19. Contour plot of the probe's receiving pattern, amplitude for openended waveguide. 
OPEN-END WAVEGUIDE SPECTRUM, PHASE

5 DEGREE CONTOURS $(60,110)$

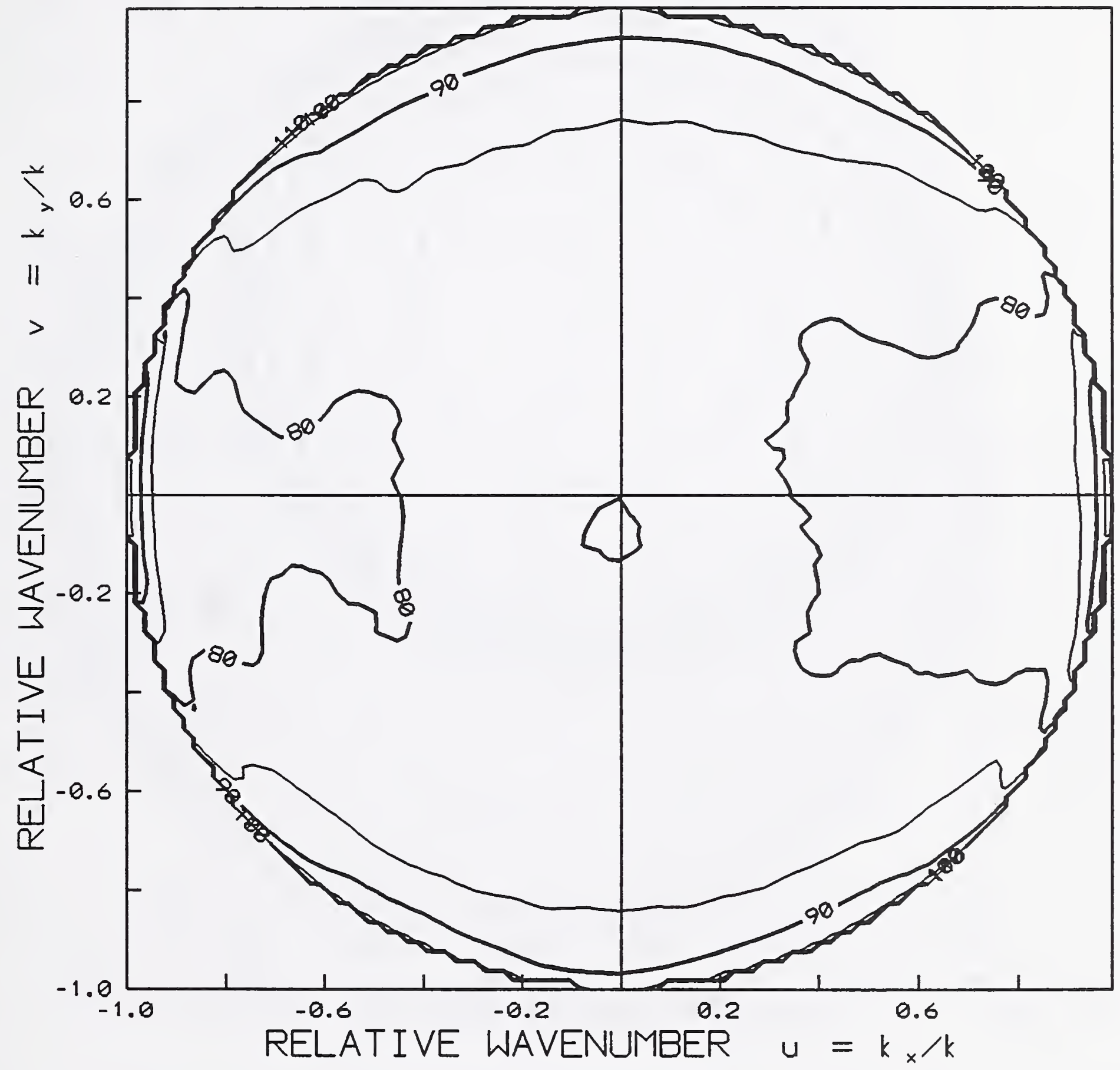

Figure 20. Contour plot of the probe's receiving pattern, phase for openended waveguide. 


\section{ELEMENT PATTERN * PROBE PATTERN - AMPLITUDE}

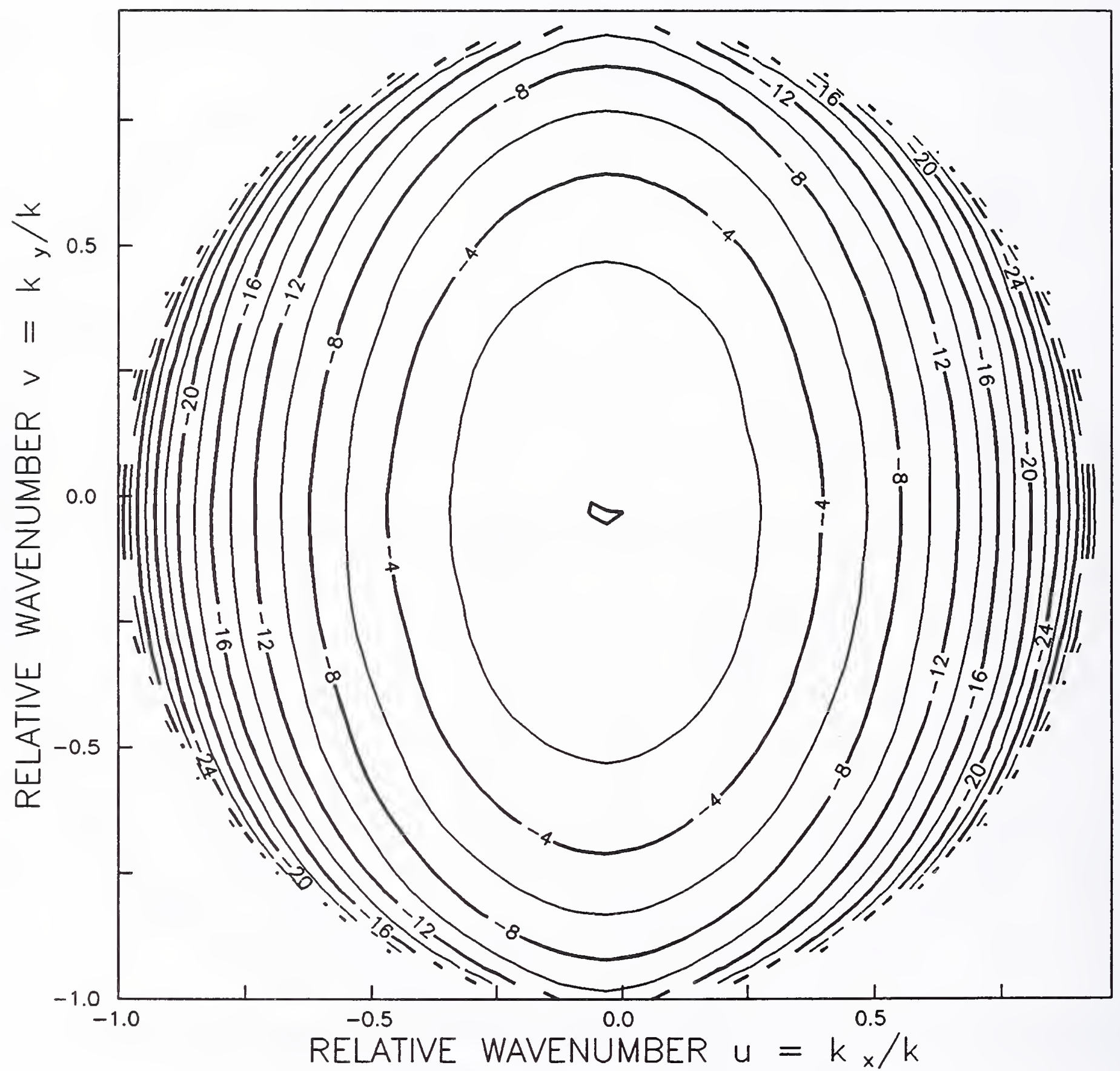

Figure 21. Amplitude of the coupling product $D^{e}(\hat{k})$ assuming probe and average element are open-ended waveguides. 


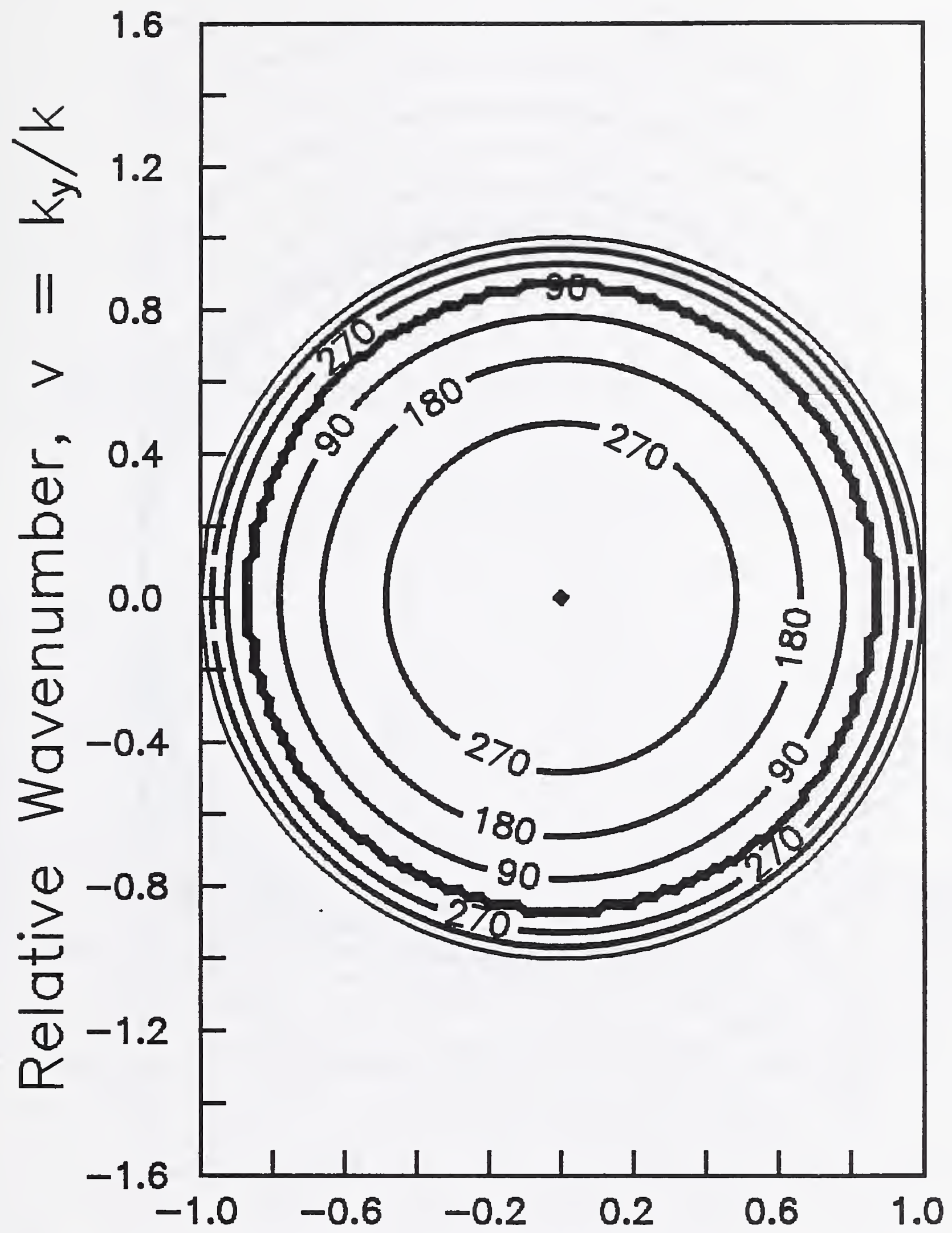

Relative Wavenumber, $u=k_{x} / k$

Figure 22. Phase of the factor $\exp (i \gamma z)$. Frequency is $3 \mathrm{GHz}$ and $z=20 \mathrm{~cm}$. 


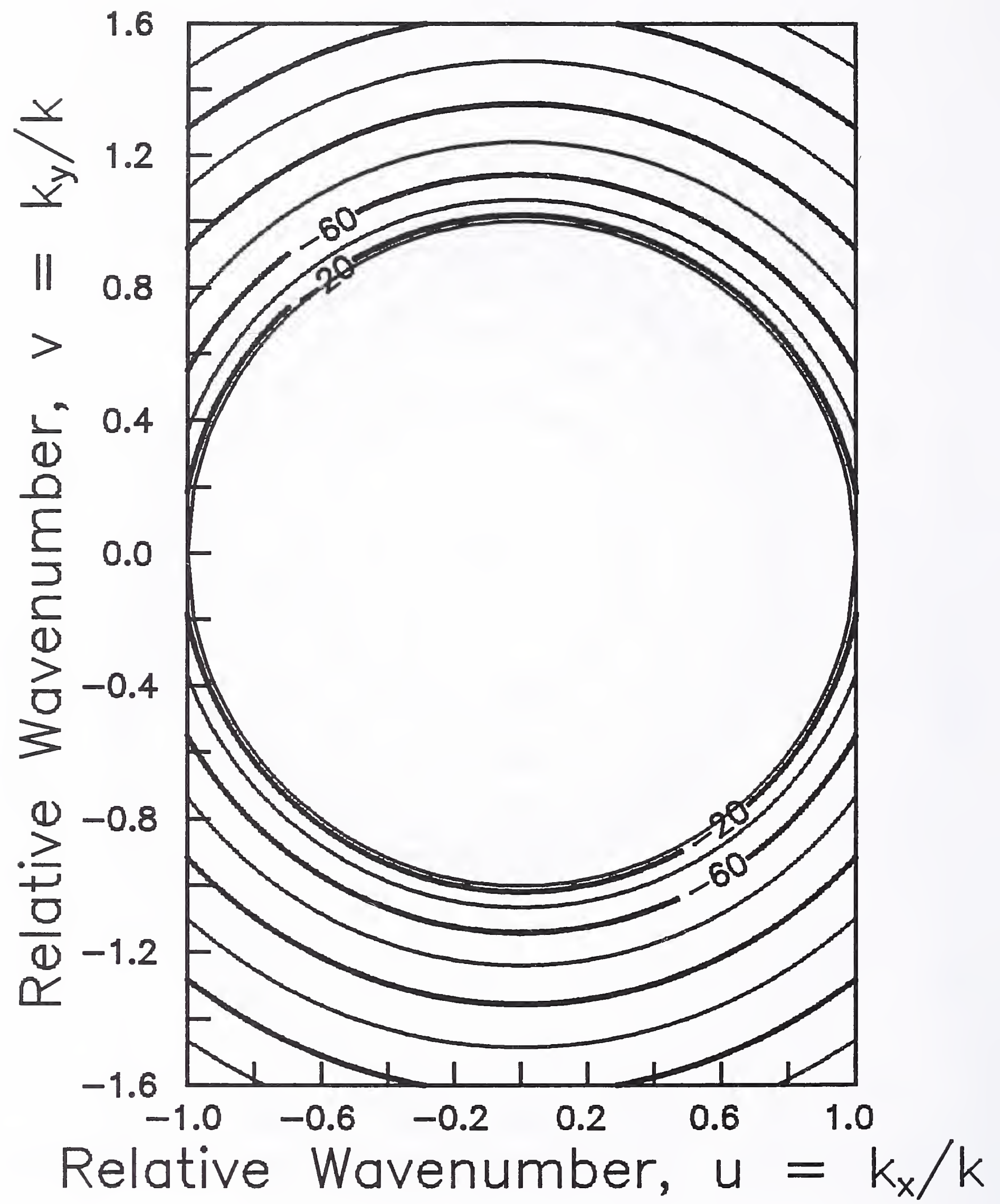

Figure 23. Amplitude of the factor $\exp (i \gamma z)$. Frequency is $3 \mathrm{GHz}$ and $z=20 \mathrm{~cm}$. 
ARRAY NEAR-FIELD AMPLITUDE. Z=20CM. NO ERRORS

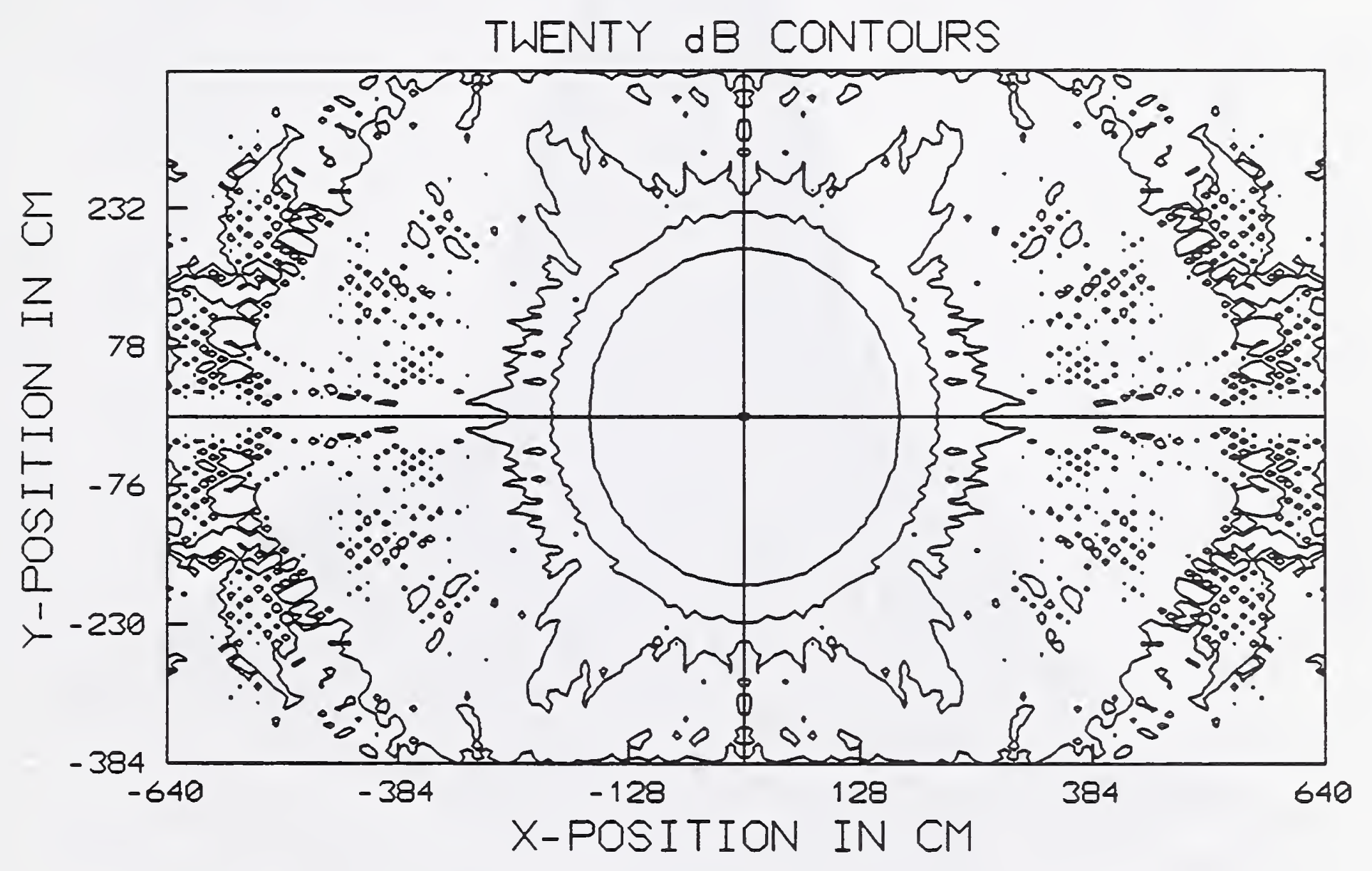

Figure 24. Simulated near-field amplitude. Frequency is $3 \mathrm{GHz}$ and $z=20 \mathrm{~cm}$. 


\section{NEAR-FIELD AMPLITDUE, Z $=20 \mathrm{CM}$}

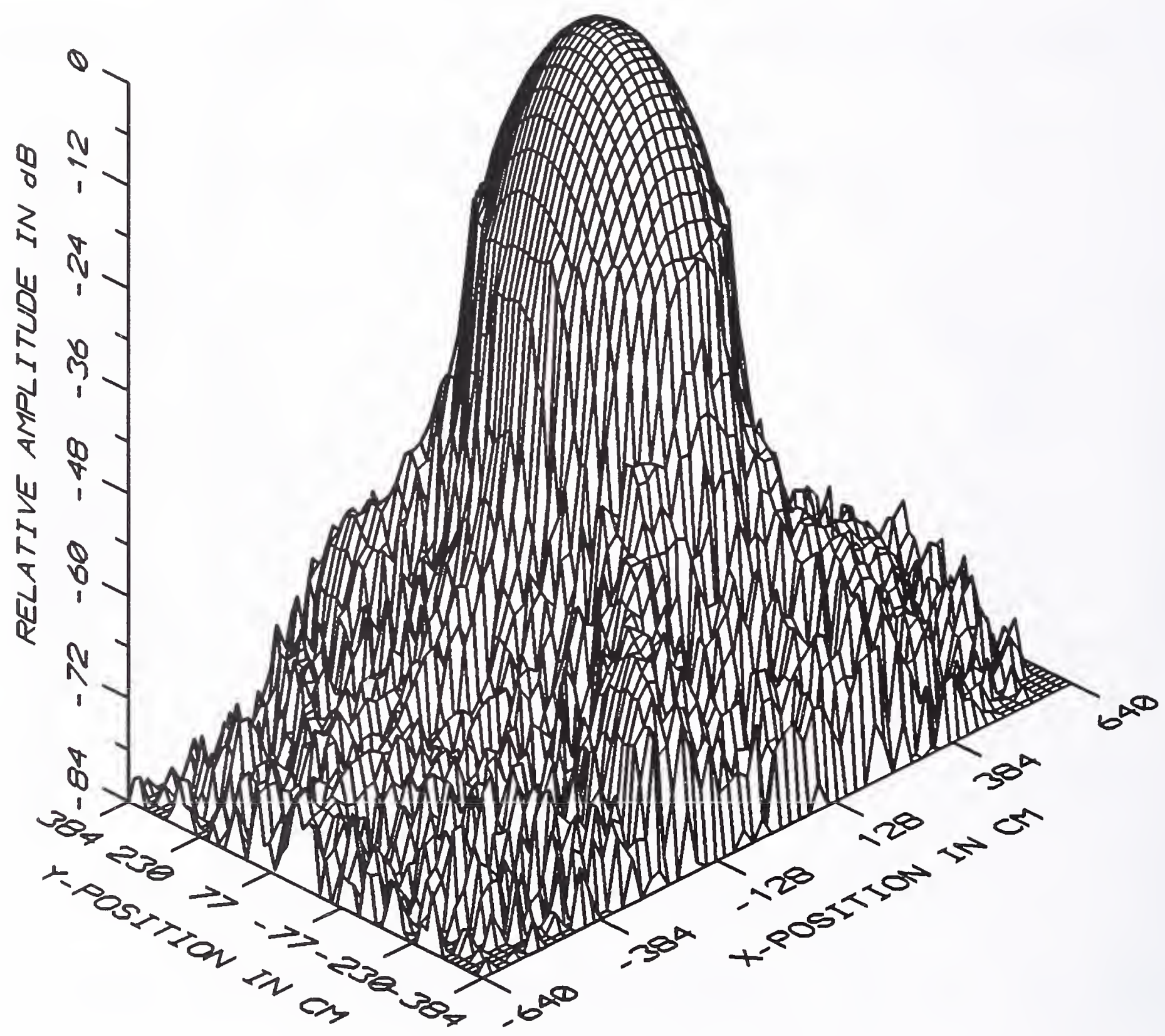

Figure 25. Perspective plot of the near-field amplitude. Frequency is $3 \mathrm{GHz}$ and $z=20 \mathrm{~cm}$. 
Simulated Near-Field Data at $z=50 \mathrm{~cm}$

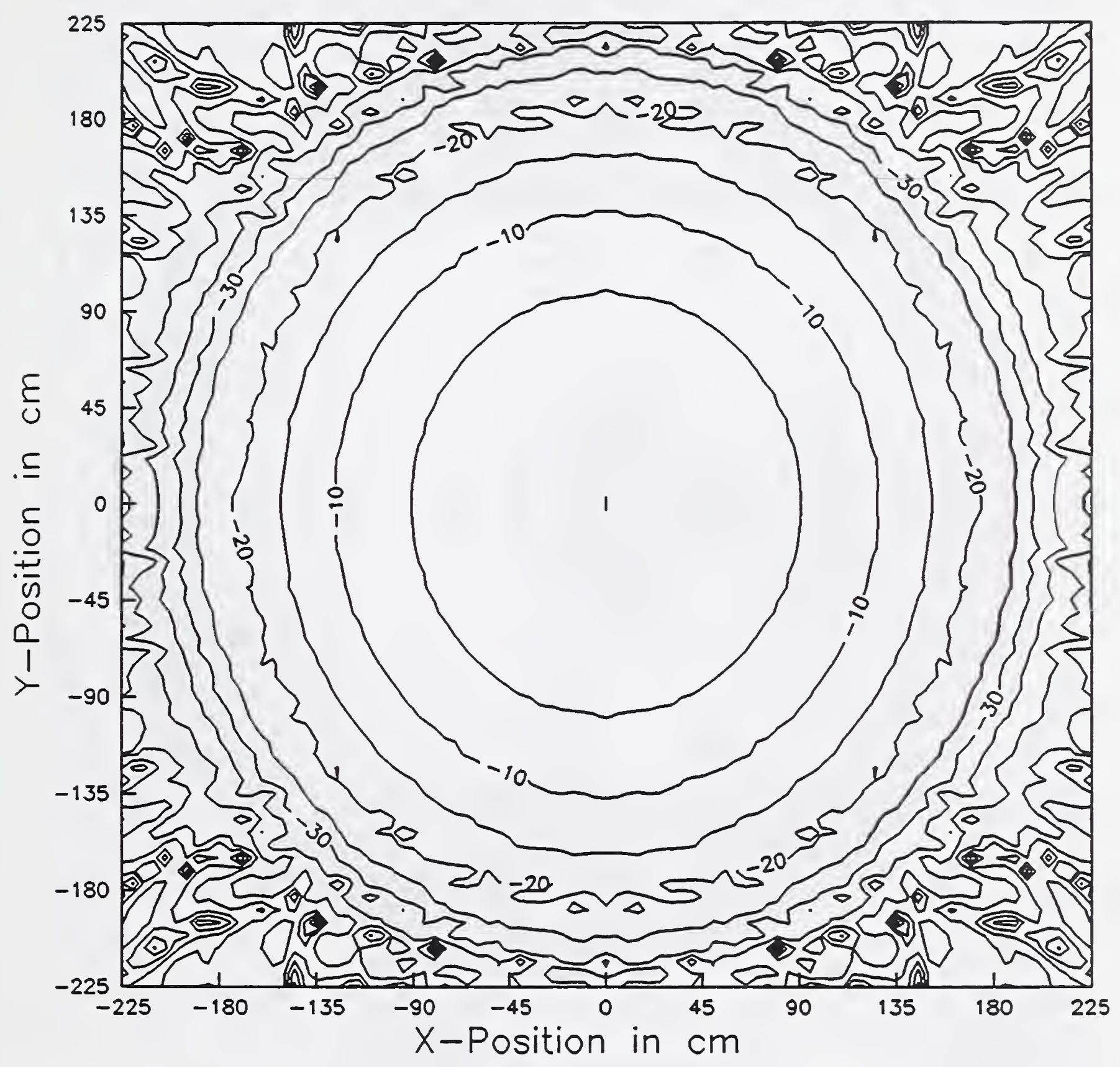

Figure 26. High resolution contour plot of the near-field amplitude near the center of the aperture. Frequency is $3 \mathrm{GHz}$ and $z=50 \mathrm{~cm}$. 
Simulated Near-Field Phase $z=50 \mathrm{~cm}$

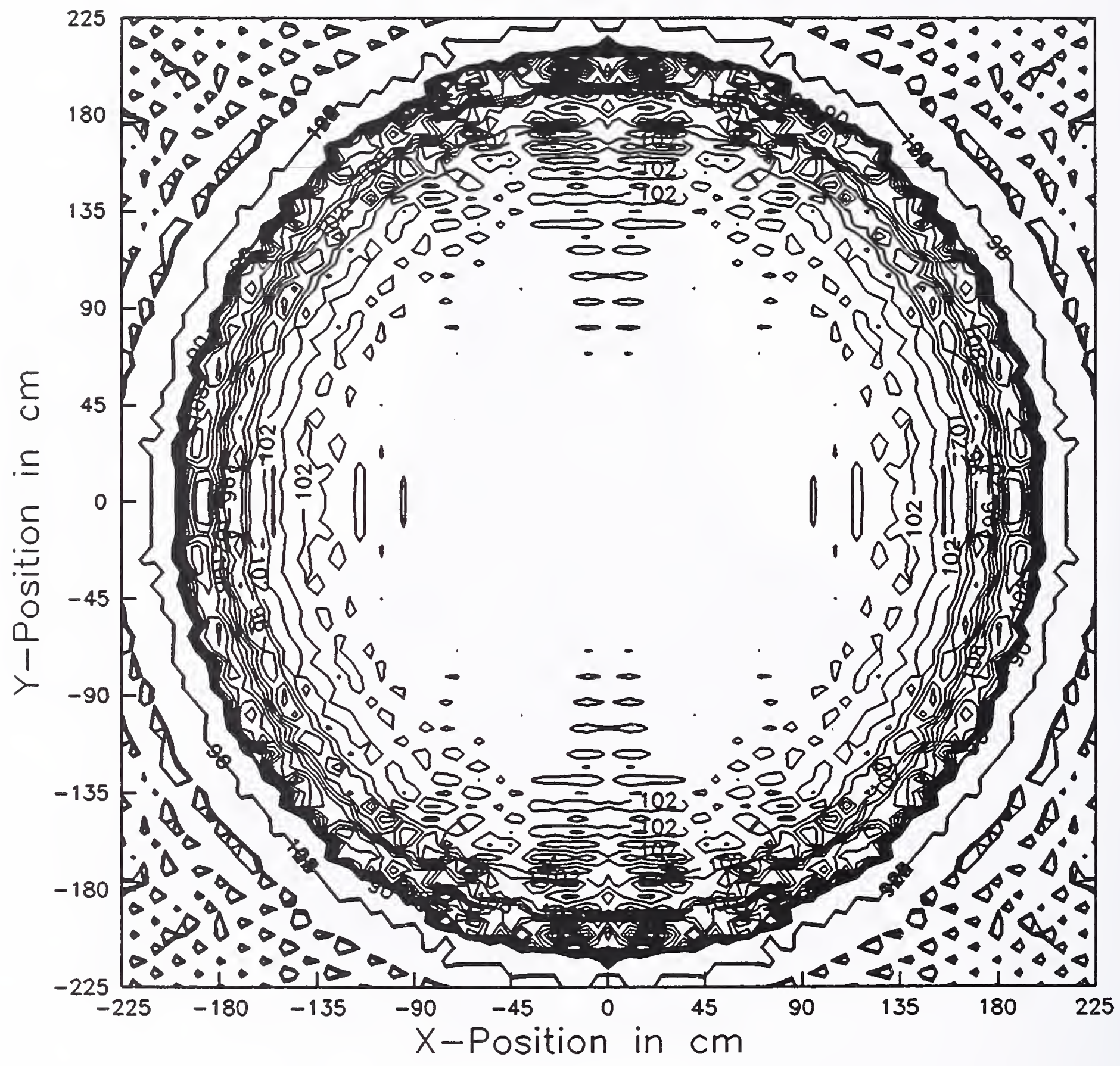

Figure 27. High resolution contour plot of the near-field phase near the center of the aperture. Frequency is $3 \mathrm{GHz}$ and $\mathrm{z}=50 \mathrm{~cm}$. 


\section{$10 \mathrm{~dB}$ Contours $(0,-60)$}

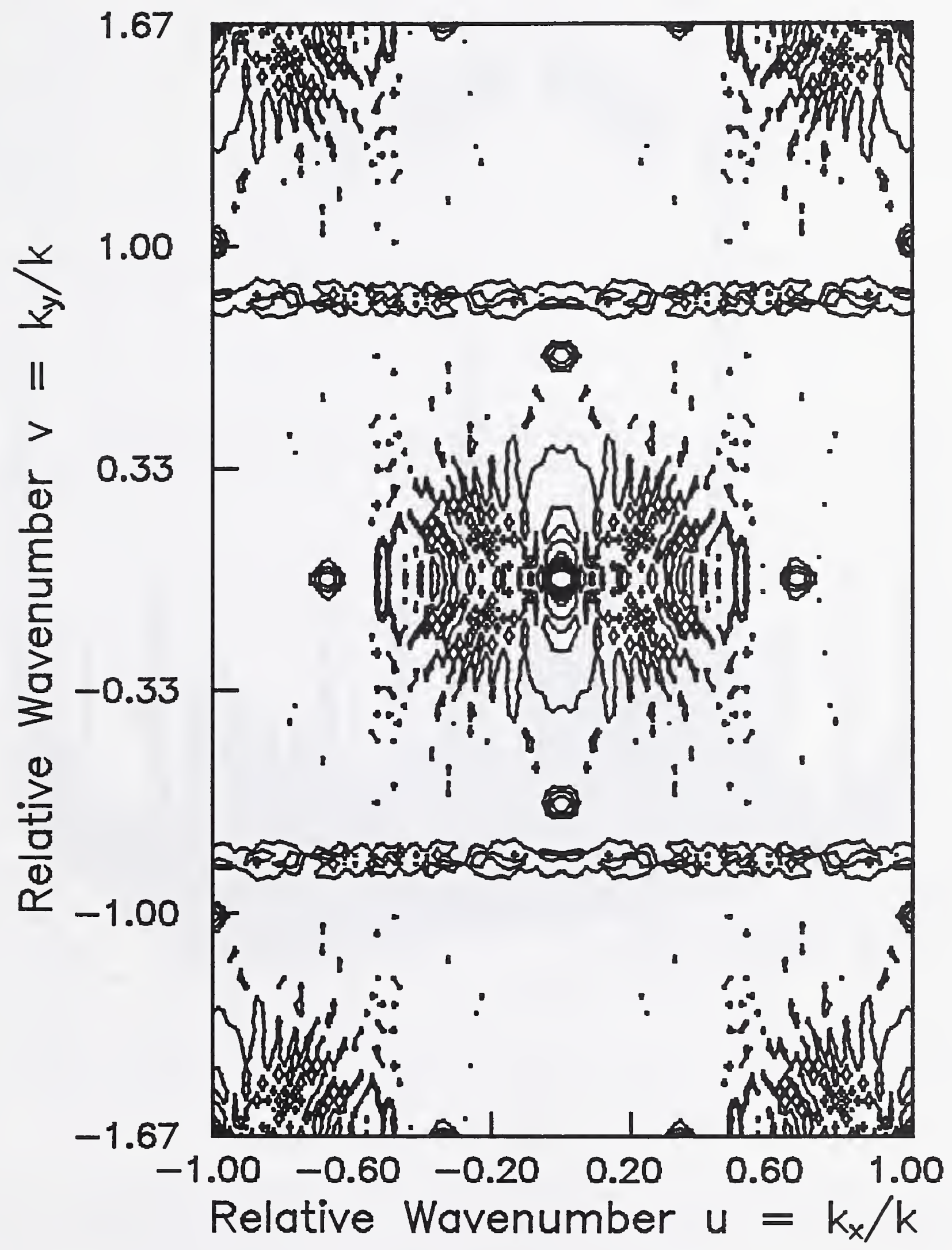

Figure 28. Contour plot of the array factor for an excitation matrix with sinusoidal phase errors in both $\mathrm{x}$ and $\mathrm{y}$ directions $\left(3^{\circ}\right.$ amplitude, $15 \mathrm{~cm}$ period). 


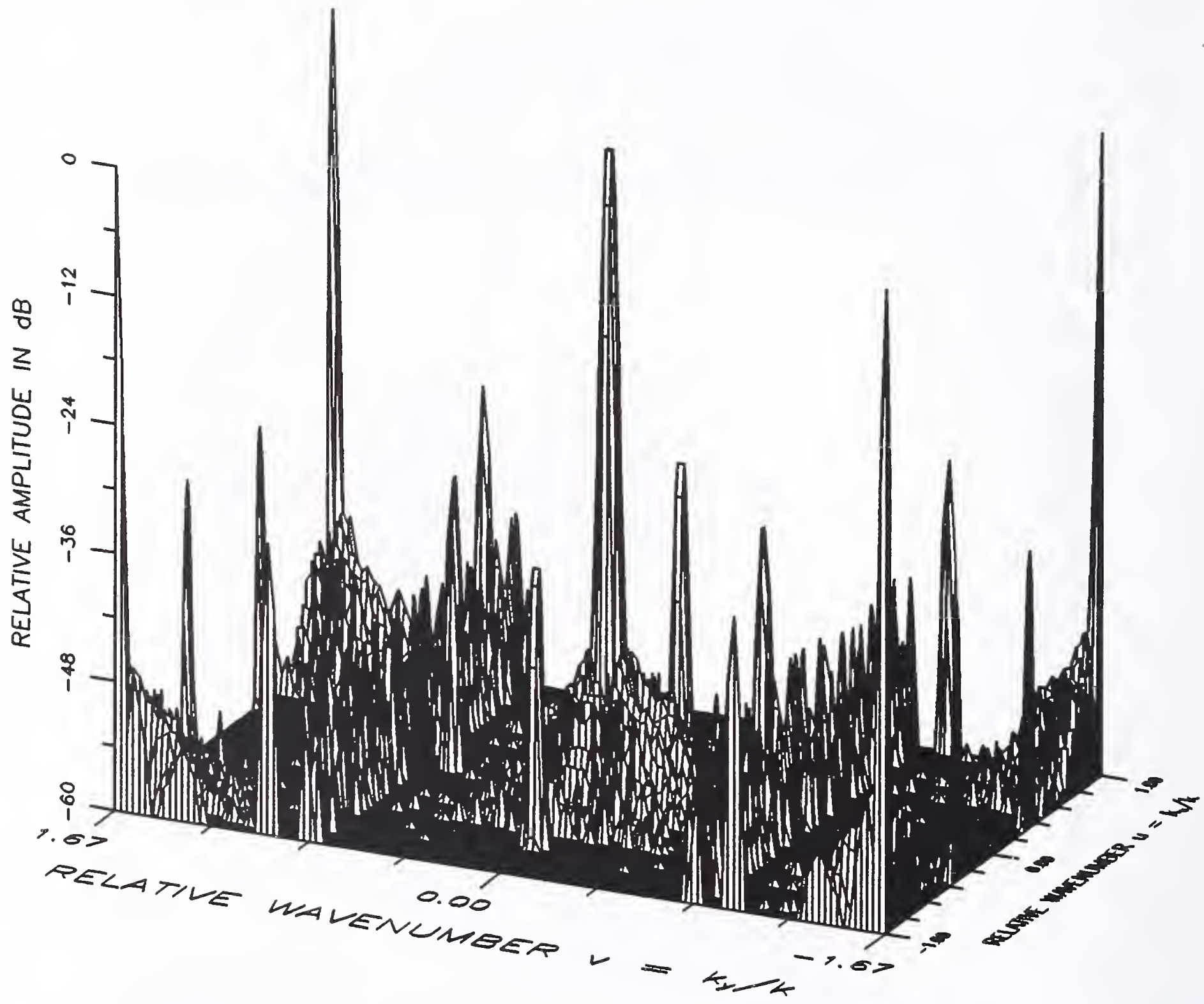

Figure 29. Perspective plot of the array factor for an excitation matrix with sinusoidal phase errors in both $\mathrm{x}$ and $\mathrm{y}$ directions $\left(3^{\circ}\right.$ amplitude, $15 \mathrm{~cm}$ period). 


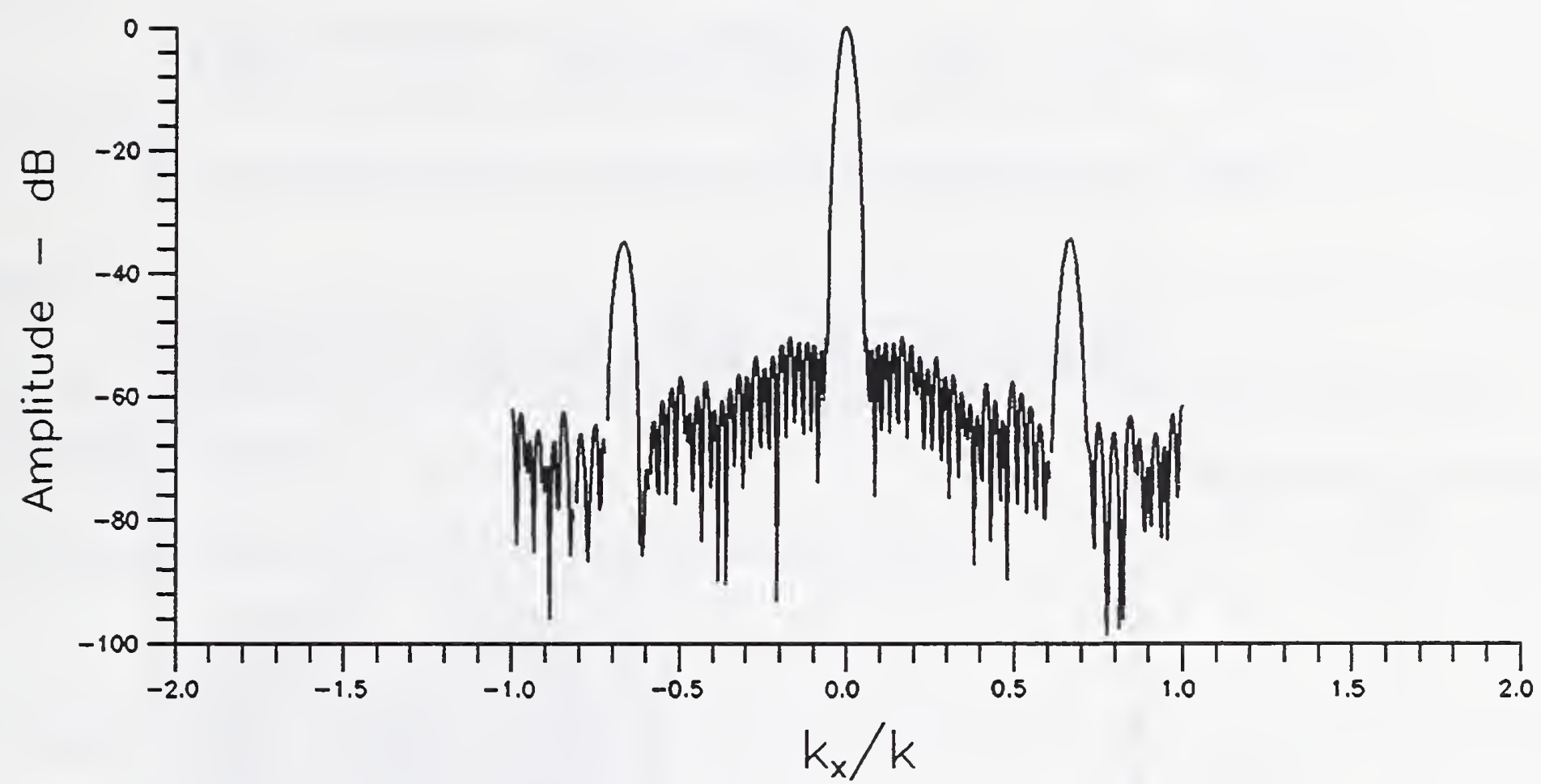

Figure 30. Principal plane $\left(k_{\mathrm{y}}=0\right)$ cut of the array factor for an excitation matrix with a sinusoidal phase error in the $x$ direction $\left(3^{\circ}\right.$ amplitude, $15 \mathrm{~cm}$ period).

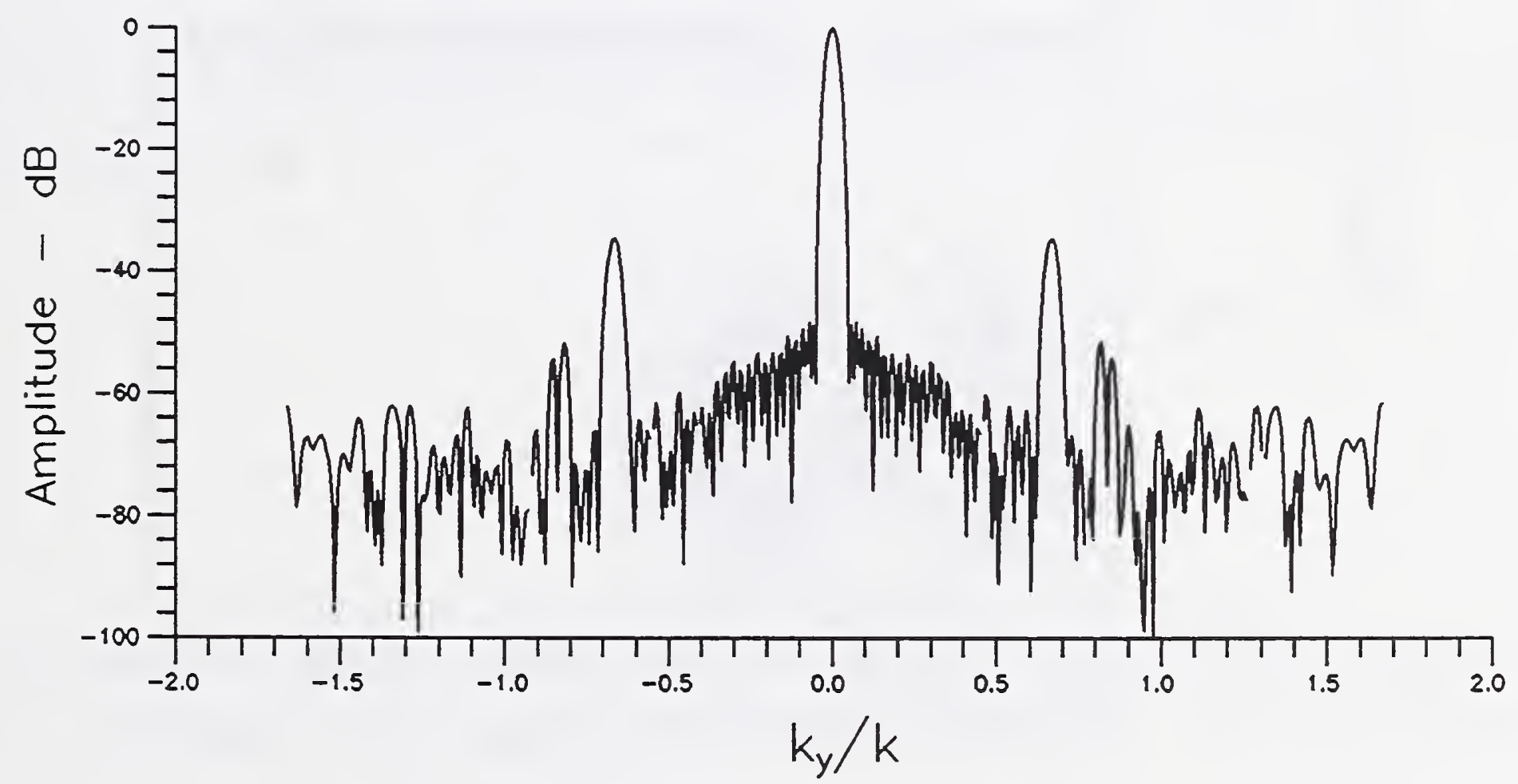

Figure 31. Principal plane $\left(k_{\mathrm{x}}=0\right)$ cut of the array factor for an excitation matrix with a sinusoidal phase error in the $y$ direction ( $3^{\circ}$ amplitude, $15 \mathrm{~cm}$ period). 
$10 \mathrm{~dB}$ Contours $(0,-60)$

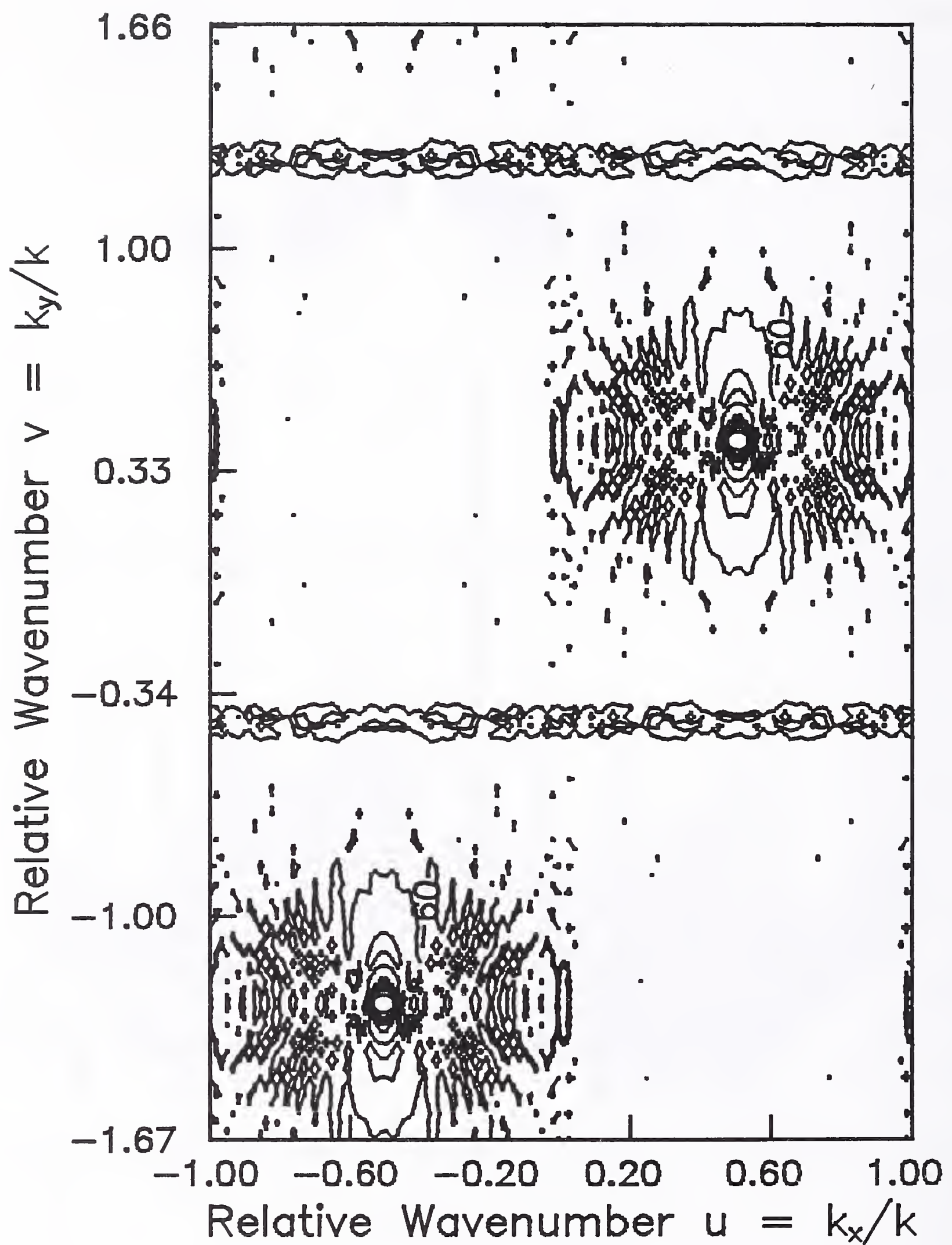

Figure 32. Contour plot of array factor for a steered beam. 


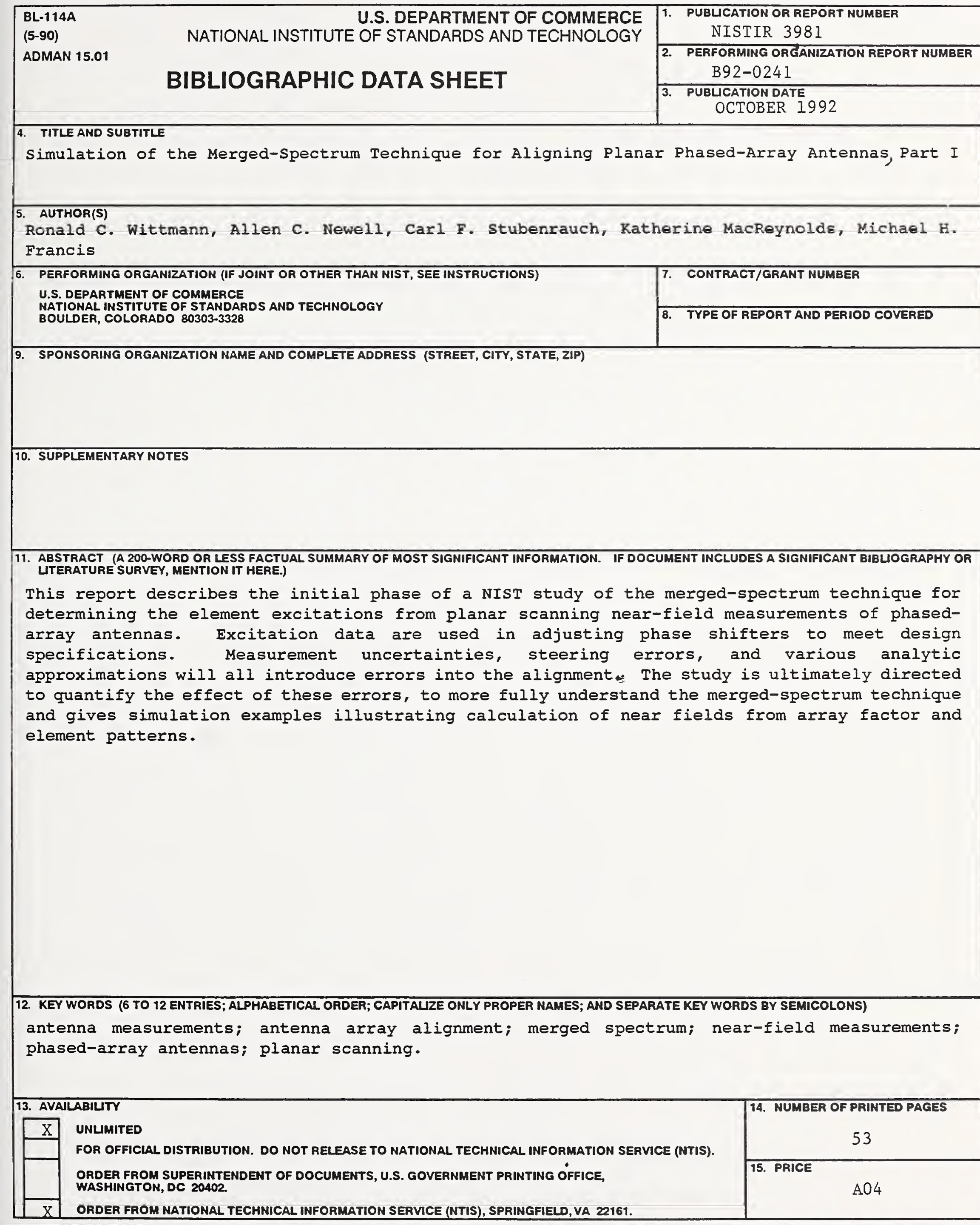






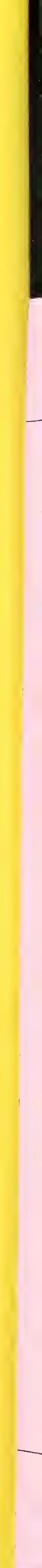

